\title{
Performance of an Adaptive Successive Serial-Parallel CDMA Cancellation Scheme in Flat Rayleigh Fading Channels
}

\author{
Tik-Bin Oon, Member, IEEE, Raymond Steele, Fellow, IEEE, and Ying Li
}

\begin{abstract}
The performance of a successive concatenated cancellation scheme for code-division multiple-access (CDMA) uplink transmission in cellular mobile radio is presented. Both serial and parallel cancellation stages are employed. The serial cancellation stage is first used to obtain initial data estimates followed by the parallel cancellation stages to enhance the accuracy of the estimates. The performance of this scheme is evaluated via analysis and simulation. In our analysis, we develop a model to consider the impact of wrongly estimating the phase and amplitude of the channel impulse response on the successive concatenated cancellation scheme. Analysis and simulation results in flat Rayleigh fading asynchronous channels with both perfect and nonperfect channel estimation and with perfect ranking confirms the accuracy of our analytical model as well as the significant improvement in performance compared to the conventional single-user matched filter (MF) detection and the stand-alone parallel cancellation schemes. Analytical results also show that the concatenated scheme has the potential to reach the single-user performance bound for a wide range of user base size, up to $K=120$ users, with a processing gain of 127 using one serial and three parallel cancellation stages. Finally, we propose a method whereby the receiver adapts with the number of users in order to retain the bit error ratio (BER) performance while decreasing the processing delay.
\end{abstract}

Index Terms-CDMA, cellular radio, interference cancellation.

\section{INTRODUCTION}

C ODE-division multiple-access (CDMA) possesses inherent flexibility to achieve a higher capacity and call quality in an interference-limited mobile radio environment compared to time and frequency multiple access systems. A conventional CDMA system employs single-user matched filter (MF) detection where the interference from other users sharing the same frequency channel is considered as Gaussian noise. This approach gives rise to the so-called near-far problem which arises when signals from mobiles arrive at base station receiver with different power levels. The consequence is that weaker signals are swamped by the stronger ones causing the

Manuscript received March 11, 1997; revised April 7, 1999. This paper was presented in part at the 47th Annual International Vehicular Technology Conference, Phoenix, AZ. This work was supported by Singapore Technologies PL, Singapore, and CVCP, U.K.

T.-B. Oon was with the Department of Electronics and Computer Science, University of Southampton, Southampton S09 5NH, U.K. He is now with CET Technologies, 609602, Singapore.

R. Steele was with the Department of Electronics and Computer Science, University of Southampton, Southampton S09 5NH, U.K.

Y. Li was with the Department of Electronics and Computer Science, University of Southampton, Southampton S09 5NH, U.K. She is now with Multiple Access Communications Limited, Southampton, SO16 7NS, U.K.

Publisher Item Identifier S 0018-9545(00)00632-0. detection of weaker signals to be unreliable. The result is a decrease in the system capacity. Also, due to the asynchronous nature of the uplink channel, the nonzero cross correlation between the users' spreading codes produces a multiple-access interference (MAI) noise floor that increases linearly with the number of users. Schemes have since been proposed to overcome the two undesirable features [1], [2]. They are, however, more complex than the single-user MF detection scheme. Complexity varies with the number of users from exponential for the optimum detection scheme proposed by Verdu [3], to lesser complexity suboptimum arrangements, such as parallel or serial successive cancellation schemes [4].

We are concerned here with CDMA communications in cellular radio where there is a limit on the maximum attainable capacity on the reverse link [5]. This is because of the uncancelled intercellular interference and the uncancelled intracellular interference. The uncancelled intracellular interference arises from the unprocessed multipath signals of the other users in the same cell. These unprocessed multipaths, which are due to frequency selective fading, being uncancelled, result in the intracellular interference. Using the approximate power control capacity formula [6] the maximum capacity gain is 2.8 when all the intracellular interference is eliminated. However, this gain decreases to 1.5 if only $50 \%$ of the other user signal energies in the same cell are captured by the RAKE receiver.

In view of the limited capacity gain, it is prudent to adopt suboptimum schemes of lesser complexity which not only combat the two major problems relating to single-user conventional MF detection, but also improve receiver performance in the MAI environment. One such scheme is the successive serial cancellation technique which was first suggested by Viterbi [6]. In this scheme, the strongest signal is first demodulated, followed by reencoding and remodulation. This signal is then subtracted from the delayed composite signal that arrived at the antenna. This difference signal is then used for the detection of the second strongest signal. The process is repeated until the weakest user signal is obtained. Further work in this area has since been done [5], [7], [8]. The approaches either utilize successive serial cancellation, or successive parallel cancellation. With parallel cancellation, at each parallel stage all the other interfering signals are cancelled simultaneously prior to the detection of the desired signal. The outputs of each parallel stage are then remodulated and subtracted from the delayed signal that arrived at the antenna, i.e., the delayed composite signal. Each technique has it own merits. Serial successive cancellation has good near-far resistance and good acquisition and tracking performance in an 


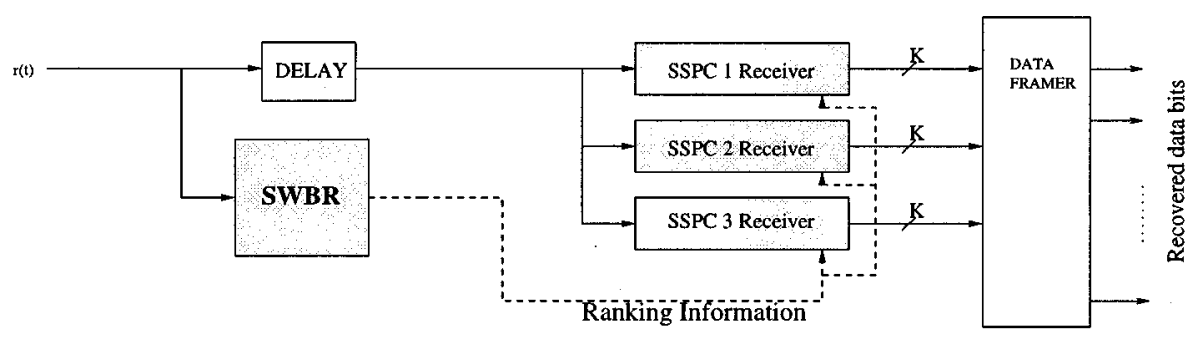

SWBR: Sliding Window Block Ranker

Fig. 1. Successive serial-parallel cancellation scheme. The data framer frames the demodulated bits from the parallel stages into respective user bit streams.

MAI channel, but suffers from decoding delay penalty. The parallel technique has less delay, but there are difficulties in code acquisition and reasonably good accurate control of the transmitted power of the mobiles is required. An extension of the successive cancellation scheme employs successive serial cancellation scheme for data detection and parallel successive cancellation technique for subsequent cancellation of the interference [9]. In this way, the strengths of both serial and parallel cancellation schemes are jointly utilized to tackle MAI induced performance degradation and thereby achieve an order of magnitude improvement in bit error ratio (BER) performance.

In Section II, we describe a SSPC scheme which is an extension of the schemes described in [4] and [9]. It consists of a sliding window block ranker and both serial and parallel cancellation stages that operate in tandem. For the serial cancellation stage, our scheme differs from the one in [4] and [10]. Instead of executing a block ranking based on the average amplitude values of $n$ bits and subsequent cancellation of these $n$ data bits, our ranking and detection outputs only consider the middle bit of a 3 -b data frame. This is done using a sliding window ranker employing a 3-b frame length and a cancellation frame of similar size. The 3-b frame size is determined to be the most cost effective in terms of BER performance and processing complexity [11]. Section III is concerned with developing a mathematical model of the SSPC scheme that accounts for both phase and amplitude channel estimation errors as well as the impact of propagation errors. As such, our deliberations differs from those in [4] and [9] with respect to the mathematical model used and the channel adopted, respectively. The mathematical model developed in [4] does not consider channel estimation errors nor error propagations. The simulation and analytical results are presented in Section IV. We will show that the serial and parallel cancellation stages operating in tandem are effective for large number of users, whereas for lightly loaded systems the serial cancellation stage alone suffices. In the same section, performance comparison will also be made with the stand alone multistage parallel cancellation (MSPC) scheme operating in similar conditions. The impact of extending the cancellation frame size and nonperfect power ranking are also examined in the subsequent sections. Section V proposes an adaptive successive serial parallel cancellation scheme where not all the users are processed by the serial cancellation stage when the number of users exceeds a system parameter $K_{T}$. By this recourse the delay is decreased while maintaining the same average probability of bit error. The final section contains the concluding remarks.

\section{SuCCESSIVE SERIAL-PARALlel CANCELlation SCHEME}

\section{A. System Overview}

The block diagram of the base station (BS) employing a successive serial-parallel cancellation scheme is shown in Fig. 1. It has three major components: the sliding window block ranker (SWBR), a bank of successive serial-parallel cancellation (SSPC) receivers each having serial and successive parallel stages, and a data framer. Based on the ranking information, the user channel having the highest signal level is processed first in the serial cancellation stage.

\section{B. Sliding Window Algorithm}

In order to achieve ranking of each user's successive data bits, the SWBR processes the received composite signal using a sliding window. The SWBR provides the ranking information based on the center bit of a 3-b window that slides one bit at a time. Fig. 2 shows that the sliding window spans three bits, and successive bits for users 1,2 , and $K$. The first window, which is shaded, only covers the users' data bits denoted by As, Bs, and Cs. Once the ranking information is obtained with respect to bit B of each user the window slides forward by one bit. The second window only covers A's, As, and Bs data bits of all the users. The ranking information is now obtained with respect to the center bit A of each user. This process then repeats for each successive data bit of each user.

Likewise, the detection and cancellation processes in both the serial and parallel stages are performed on a 3-b frame basis that corresponds directly to the 3-b frame used in the sliding window ranking process. Note that, although, the detection and cancellation process is done on a 3-b frame, only the data bits associated with the center bit of the frame are generated at the output of the final parallel stage. In other words, each SSPC receiver executes both sequence detection and cancellation encompassing a length of three bits which corresponds to the 3-b frame in the SWBR process. The first SSPC receiver in Fig. 1 processes all the users' data bits corresponding to the first window, while the third SSPC receiver processes all the users' data bit that corresponds to the third window while the fourth window is then processed by the first SSPC receiver, and so on. In this way, each successive bit of each user is processed. The frame length of three bits is necessary to cover the asynchronous interaction between the users' data bits. To see this, let us refer to the first window of Fig. 2. Assuming that the second, first, and $K$ th user's signal is the strongest, second strongest, and the weakest, 


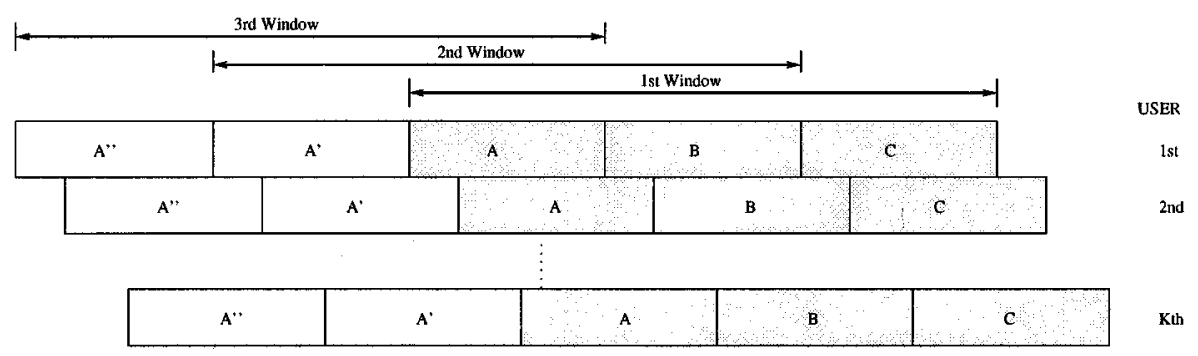

Data Stream

Fig. 2. Sliding windows process in asynchronous channel.

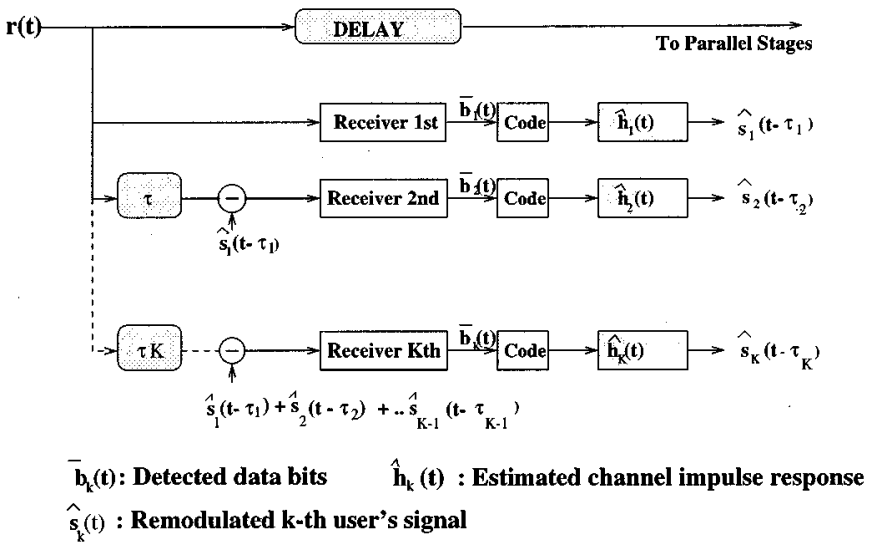

Fig. 3. Successive serial cancellation stage (SSC).

respectively. In this scenario, the $\mathrm{A}$ and $\mathrm{C}$ bits, corresponding to the second user's signal, are affecting the center bit (i.e., bit B) of the first and the $K$ th users' signals, respectively. If only bit A or $\mathrm{C}$ of the strongest signal is cancelled, strong residual MAI on other users' data bits would still be present. The configuration depicted in Fig. 1 assumes that hardware delay lines are used to delay the bandpass signal $r(t)$. If the composite signal after down conversion, is first digitized and stored in memory, only a single equivalent SSPC receiver is required to process the baseband signal.

In the following discussion on detection and cancellation processes we will assume, for ease of exposition that the first user is the strongest user, followed by the second who is the next strongest, and so on.

\section{Successive Serial Cancellation Stage}

The successive serial cancellation stage (SSC) in the SSPC receiver uses successive serial cancellation to obtain reliable data estimates for interference regeneration. The strongest user signal identified by the SWBR is first CDMA detected to give the 3-b sequence $\bar{b}_{1}(t)$ in Fig. 3. To improve the estimate of the bits sequence $\bar{b}_{2}(t)$, associated with the second user, we attempt to remove the interference due to the strongest signal on a 3-b frame basis as indicated earlier. Thus, the 3-b sequence denoted by $\bar{b}_{1}(t)$ is CDMA coded and convolved with the estimates of the channel impulse response $\hat{h}_{1}(t)$ to give $\hat{s}_{1}\left(t-\tau_{1}\right)$.
We now have an estimate of the CDMA signal that arrived at the BS receiver for the strongest user. We see in Fig. 3 that we need to allow an appropriate delay $\tau$ to $r(t)$ to allow for the processing delay due to the cancellation process before subtracting $\hat{s}_{1}\left(t-\tau_{1}\right)$ from it. This delay $\tau$ is determined by the time required to demodulate the three data bits and regenerate the corresponding signal. A CDMA signal is now formed which is essentially devoid of the strongest interference component, namely, the interference from user 1 . The second CDMA receiver in the SSC operates on $r(t-\tau)-\hat{s}_{1}\left(t-\tau_{1}\right)$ to give a better estimates of data bits, namely, $\bar{b}_{2}(t)$. Note that $\tau_{1}$, and the more generally $\tau_{k}$, relates to the delay of the CDMA signal over a users' radio channel. Note also that, only one bit in this 3-b block sequence denoted by $\bar{b}_{2}(t)$, is the desired bit and subsequently produced at the final parallel stage. Once again we CDMA code $\bar{b}_{2}(t)$ and pass it through our estimate of second user's channel to give another CDMA signal $\hat{s}_{2}\left(t-\tau_{2}\right)$. Now $\hat{s}_{2}\left(t-\tau_{2}\right)$ is subtracted from $r(t-2 \tau)$ and the user ranked third by the SWBR is used to recover bits $\bar{b}_{3}(t)$, and so on. This process is repeated until the weakest signal is detected. This SSC process could be viewed as a serial cancellation front-end to the parallel cancellation stages in the next subsystem block.

This detection and cancellation procedure starting with the strongest received CDMA signal and progressing to the weakest received signal has several advantages. For example, it is easier to acquire and track the strongest signal, and the data detected and subsequent cancellation is more reliable leading to less error propagation.

\section{Successive Parallel Cancellation Stage}

Having regenerated a set of estimated and separable CDMA signal $\hat{s}_{j}\left(t-\tau_{j}\right) ; j=1,2, \ldots K$, for the $K$ users, we continue to seek an improvement to these estimates. This is achieved by deploying a sequence of successive parallel cancellation stages (SPC's). Fig. 4 shows the first stage. The received signal $r(t)$ delayed by a further $\tau K$ is applied to the input of the first SPC. For the first user, we subtract all the interfering CDMA estimates from $r(t)$ to give an improved estimate of $s_{1}(t)$ which is CDMA decoded to give bits $\bar{b}_{1}^{1}(t)$. The same process is repeated for all the other users. If no further stage is required, the center bit of the 3-b block $\bar{b}_{k}^{1}(t)$ for each user is sent to the data framer. 


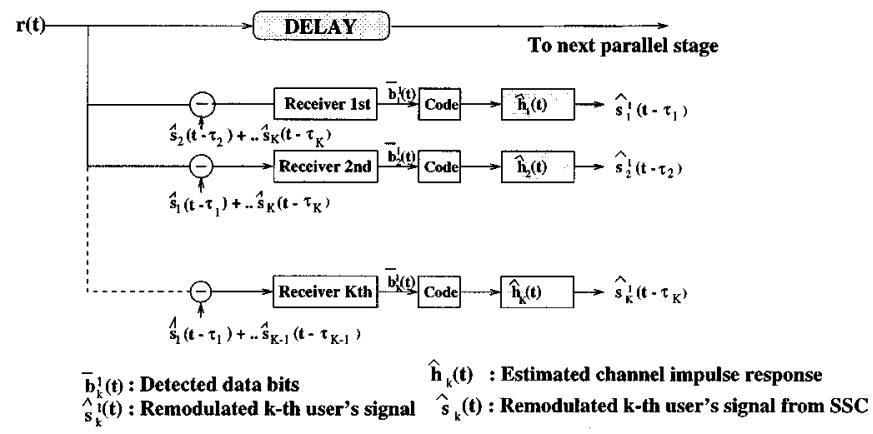

Fig. 4. Successive parallel cancellation stage (SPC).

The purpose of the data framer is to frame the output bits into respective user's demodulated bit stream. This is needed as the rank of each user's signal in a 3-b block changes from block to block. The changes in the ranking is a function of the Doppler fading rate of the channels.

If additional parallel cancellation stages are required, the CDMA signal corresponding to the 3 -b block $\bar{b}_{k}^{1}(t)$ is regenerated for each user. This set of regenerated CDMA signals $\hat{s}_{j}^{1}\left(t-\tau_{j}\right) ; j=1,2, \ldots K$, for the $K$ users are then applied to the input of the second SPC. As before, for the first user, we subtract all the interfering CDMA estimates from an appropriately delayed $r(t)$ to give a further improved estimate of $s_{1}(t)$ which is CDMA decoded to give bits $\bar{b}_{1}^{2}(t)$, and so on. This process is repeated for the next successive SPC, if required.

As $\hat{s}_{j}\left(t-\tau_{j}\right) ; j=1,2, \ldots K$, are asynchronous there is MAI from parts of other users bits. Thus, in generating $\bar{b}_{k}^{1}(t)$ we use a window of sufficient width such that if the center bit of $\bar{b}_{k}^{1}(t)$ is bit B in Fig. 2, we consider the interference from bits $\mathrm{A}, \mathrm{B}$, and $\mathrm{C}$ from the other users within the same window, as mentioned earlier. Notice that our process only covers three bits interaction between the user's signal. However, due to the asynchronous nature of the CDMA channel, the two overlapping bits of the interferer's signal on the desired bit are also themselves subjected to interference arising from other bits of other users' signals which are wider than the 3-b frame width. So, if a larger cancellation frame is adopted, the MAI on the desired bit can be decreased resulting in a lower average probability of bit error.

The flowchart in Fig. 5 summarizes the complete SSPC process as described earlier. To recapitulate, the SSPC process begins with ranking, this is followed by successive serial detection, interference regeneration of the strongest signal and the cancellation of this strongest signal from the composite received signal $r(t)$ on a 3-b basis. This process is repeated for each user's signal until the weakest user bit is decoded. After the serial stage of detection and cancellation are completed, the regenerated interference signals of the serial stage are used in the first parallel cancellation stage. In the parallel cancellation stages, the process of interferences cancellation, demodulation, and interferences regeneration are done in a parallel format.

\section{AnAlytical Model of the SSPC Scheme}

In this section, we will consider the analytical model of the the SSPC receiver. We first derive the model for the serial stage, follow by the derivation of the complete model. In so doing,

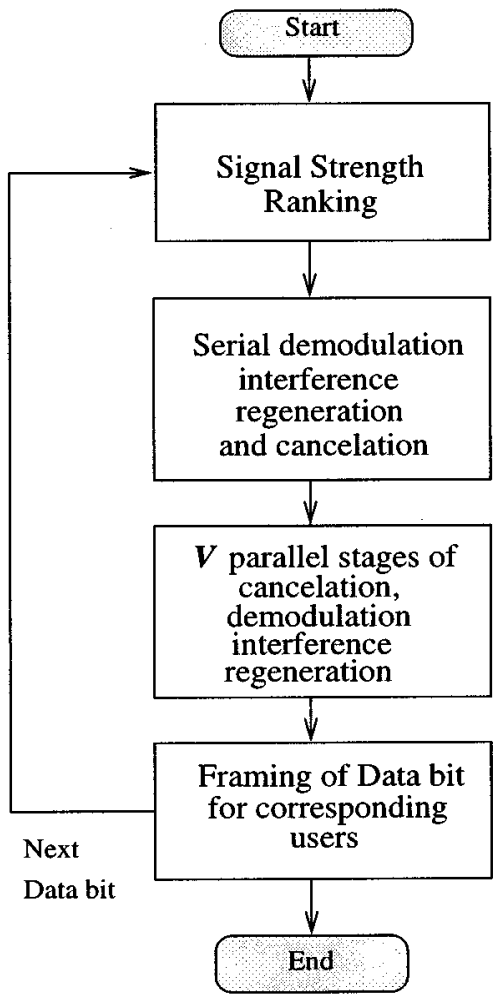

Fig. 5. Successive serial-parallel cancellation algorithm.

we shall adopt the technique employed in [12] to derive the bit error probability. We first obtain the ranked conditional probability of bit error at the output of each MF receiver in the serial stage and at the outputs of the bank of MF receivers in each of the parallel stages. The conditioning, which is based on the ranked Rayleigh-distributed amplitude of the received signal, is then removed by averaging the conditional bit error probability with the appropriate probability density function (PDF) over the range of likely ranked values.

\section{A. Assumptions}

In our analysis, we only consider the reverse channel of a single-cell CDMA cellular system which is depicted in Fig. 6. We assume that there is perfect open-loop power control, ${ }^{1}$ the BS knows the spreading Gold code sequences of each mobile station (MS), and there is perfect carrier and code phase synchronization. At the $k$ th MS transmitter, each data bit of the $k$ th user's data sequence $b_{k}(t)$ is multiplied by a Gold code sequence, $c_{k}(t)$. The pulse shapes, $\Lambda_{T}(t)$ of the data and Gold code signals are rectangular and the respective signals are

$$
b_{k}(t)=\sum_{n=-\infty}^{\infty} b_{n}^{k} \Lambda_{T}(t-n T)
$$

and

$$
c_{k}(t)=\sum_{n=-\infty}^{\infty} c_{n}^{k} \Lambda_{T}\left(t-n T_{c}\right)
$$

\footnotetext{
${ }^{1}$ This is only to facilitate easier analysis as the serial cancellation scheme performs better in an unequal power situation [9].
} 


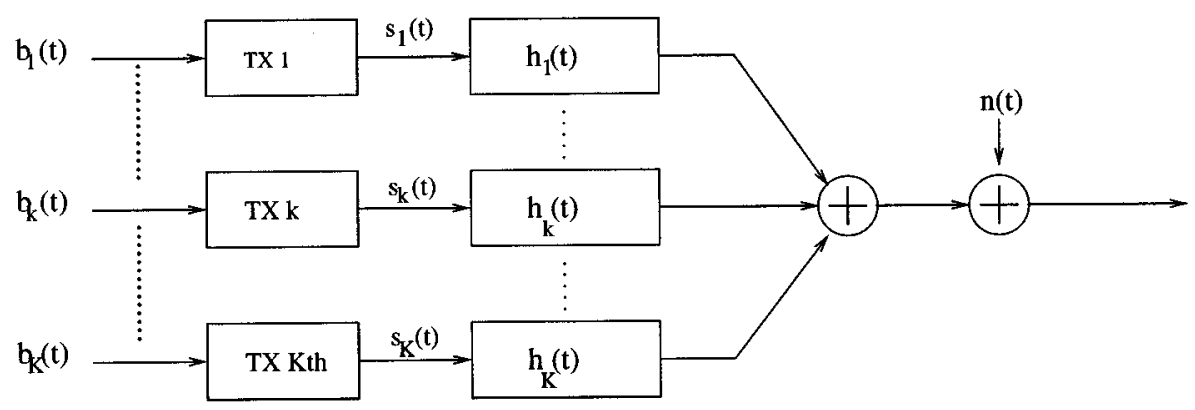

Fig. 6. Transmitter model.

where $b_{n}^{k}$ is the $n$th data bit of the $k$ th user having values of \pm 1 with equal probability, $T$ is the data bit period, $c_{n}^{k}$ is the $n$th chip of the $k$ th user having the values of $\pm 1, T_{c}$ is the Gold code chip period, and $T=N T_{c}$. There are $N$ chips in one bit interval and this is also known as the processing gain. The BPSK signal of each MS transmitter is

$$
s_{k}(t)=\sqrt{2 P_{k}} b_{k}(t) c_{k}(t) \cos \left(\omega_{c} t+\theta_{k}\right)
$$

where $P_{k}$ is the average transmitted power and $\theta_{k}$ is the carrier phase and $\omega_{c}$ is the carrier frequency in radians per second.

\section{B. Channel Model}

For a flat Rayleigh fading channel the baseband channel impulse response for the channel used by the $k$ th $\mathrm{MS}$ is

$$
h_{k}(t)=\alpha_{k} \delta\left(t-\tau_{k}\right) \exp \left(j \Phi_{k}\right)
$$

where $\alpha_{k}$ is the Rayleigh-distributed amplitude, $\tau_{k}$ is the associated asynchronous delay with uniform distribution between $[0, T)$, and $\Phi_{k}$ is the associated phase with a uniform distribution between $[0,2 \pi)$. The channel is assumed to be slow fading with parameters that are time invariant over one bit period. In the analysis of the serial stage, we do not address the impact of imperfect ranking. We model the channel estimation errors for both phase, $\triangle \phi_{k}$, and amplitude, $\triangle \alpha_{k}$, as independent and identically distributed zero mean Gaussian random variables with variances $\sigma_{\triangle \phi_{k}}^{2}$ and $\sigma_{\triangle \alpha_{k}}^{2}$, respectively. The estimated amplitude and phase of the $k$ th channel is therefore

$$
\begin{gathered}
\hat{\alpha}_{k}=\alpha_{k}+\triangle \alpha_{k} \\
\hat{\phi}_{k}=\phi_{k}+\triangle \phi_{k} .
\end{gathered}
$$

Following the approach adopted in [13], the respective variances of the estimation errors are defined as

$$
\begin{aligned}
& \sigma_{\triangle \alpha_{k}}^{2} \triangleq E\left[\left(\triangle^{2} e\right)\right] \\
& \sigma_{\triangle \phi_{k}}^{2} \triangleq \pi^{2} E\left[\left(\triangle^{2} e\right)\right]
\end{aligned}
$$

where $E\left[\left(\triangle^{2} e\right)\right]$ is the mean square error. Each channel has the same amount of estimation error in terms of the mean square error (MSE). The average MAI noise is modeled as a zero mean Gaussian random variable, and the Gaussian approximation technique is used to evaluate the variance [14]. We will show that this technique is sufficiently accurate for most cases where imperfect channel estimations prevail.

\section{SSC Stage Model}

The received signal at the base station is the sum of the received CDMA signals from $K$ users, viz.

$r(t)=\sum_{k=1}^{K} \alpha_{k} \sqrt{2 P} b_{k}\left(t-\tau_{k}\right) c_{k}\left(t-\tau_{k}\right) \cos \left(\omega_{c} t+\phi_{k}\right)+n(t)$

where $P$ is the average received power of each user, $\phi_{k}=$ $\left(\Phi_{k}+\theta_{k}-\omega_{c} \tau_{k}\right)$, is the $k$ th user random phase with uniform distribution between $[0,2 \pi)$, and $n(t)$ is the zero mean additive white Gaussian noise (AWGN) process representing thermal noise with two-sided PSD equal to $N_{\circ} / 2$ (watts/hertz). The received power $P$ is the same for each user because of perfect open-loop power control which is regularly modifying each mobile's transmitted power. Without loss of generality we can consider the composite signal $r(t)$ as a ranked set of signals with $k=1$ as the strongest user's signal and $k=K$ as the weakest user's signal, as well as setting the respective $\tau_{k}$ to zero. At the output of the first MF receiver in the SSC stage, i.e., the signal at the input to the decision circuit, called the decision statistic $Z_{s, 1}$, is given by

$$
\begin{aligned}
Z_{s, 1} & =\int_{0}^{T} r(t) c_{1}(t) \cos \left(\omega_{c} t+\hat{\phi}_{1}\right) d t \\
& =D_{s, 1}+I_{s, 1}+\eta
\end{aligned}
$$

where the subscripts $s$ and 1 denote the serial stage and the strongest received signal, respectively, the component $D_{s, 1}$ is the desired signal of the strongest user, and $I_{s, 1}$ and $\eta$ are the zero mean Gaussian random variables due to MAI and AWGN, respectively. This decision statistic determines the strongest signal data bit estimate $\bar{b}_{n}^{1}$, according to the following decision rule:

$$
\begin{aligned}
\bar{b}_{n}^{1} & =1, \quad Z_{1} \geq 0 \\
& =-1, \quad Z_{1}<0 .
\end{aligned}
$$

The expression for $D_{s, 1}$ is

$$
\begin{aligned}
D_{s, 1} & =\int_{0}^{T} \alpha_{1} \sqrt{2 P} b_{1}(t) \cos \left(\omega_{c} t+\phi_{1}\right) \cos \left(\omega_{c} t+\hat{\phi}_{1}\right) d t \\
& =\int_{0}^{T} \frac{\alpha_{1}}{2} \sqrt{2 P} b_{1}(t)\left[\cos \left(\phi_{1}-\hat{\phi}_{1}\right)+\cos \left(2 \omega_{c} t+\phi_{1}+\hat{\phi}_{1}\right)\right] \\
& \approx \alpha_{1} T \sqrt{\frac{P}{2}} b_{1}(t) \cos \left(\phi_{1}-\phi_{1}-\triangle \phi_{1}\right) \\
& =\alpha_{1} T \sqrt{\frac{P}{2}} b_{1}(t) \cos \left(\triangle \phi_{1}\right)
\end{aligned}
$$


The double-frequency term $2 \omega_{c}$ in (13) is omitted as it is approximately zero after the integration. The MAI is given by

$$
\begin{aligned}
& I_{s, 1} \\
& =\sum_{k=2}^{K} \alpha_{k} \sqrt{\frac{P}{2}} \int_{0}^{T} b_{k}\left(t-\tau_{k}\right) c_{k}\left(t-\tau_{k}\right) c_{1}(t) \cos \left(\phi_{k}-\hat{\phi}_{1}\right) d t
\end{aligned}
$$

and the AWGN component is

$$
\eta=\int_{0}^{T} n(t) c_{1}(t) \cos \left(\omega_{c} t+\hat{\phi}_{1}\right) d t .
$$

The conditional variance of $I_{s, 1}$ is, on using the Gaussian approximation

$$
\operatorname{Var}^{\prime}\left(I_{s, 1}\right)=\frac{N T_{c}^{2}}{3} \sum_{k=2}^{K} P \alpha_{k}^{2} E\left[\cos ^{2}\left(\phi_{k}-\hat{\phi}_{1}\right)\right]
$$

In (64) of the Appendix we show that $E\left[\cos ^{2}\left(\phi_{k}-\hat{\phi}_{1}\right)\right]=\frac{1}{2}$, yielding

$$
\operatorname{Var}^{\prime}\left(I_{s, 1}\right)=\frac{N T_{c}^{2}}{6} P \sum_{k=2}^{K} \alpha_{k}^{2}
$$

The variance for the AWGN term in (10) can be shown to be

$$
\operatorname{Var}(n)=\frac{N_{o} T}{4}
$$

We are now in a position to obtain the SIR expression, which is conditioned on $\alpha_{k}$ and $\triangle \phi_{1}$, at the output of the MF in the SSC stage as

$$
\operatorname{SIR}_{s, 1}^{\prime}=\frac{D_{s, 1}^{2}}{\operatorname{Var}^{\prime}\left(I_{s, 1}\right)+\operatorname{Var}(\eta)}
$$

where

$$
D_{s, 1}^{2}=\alpha_{1}^{2} T^{2} \frac{P}{2}\left(\cos ^{2}\left(\triangle \phi_{1}\right)\right) .
$$

The probability of bit error is given by

$$
P b_{s, 1}=E\left\{Q\left(\sqrt{E\left[\operatorname{SIR}_{s, 1}^{\prime}\right]}\right)\right\}
$$

where $E[\cdot]$ is the expectation operator and the $Q(\cdot)$ function is defined as

$$
Q(x) \triangleq \frac{1}{2 \pi} \int_{x}^{\infty} \exp \left(-\frac{u^{2}}{2}\right) d u .
$$

It will be recalled from Fig. 3 that having recovered $\bar{b}_{n}^{1}$, albeit with a probability of bit error $E\left\{Q\left(\sqrt{E\left[\mathrm{SIR}_{s, 1}^{\prime}\right]}\right)\right\}$, we then re-CDMA code it and convolve it with our estimates of the channel impulse response. The emerging CDMA signal represents an estimate of the strongest component in the originally received signal $r(t)$. It is therefore the strongest interference signal to all the $K-1$ users and is given by

$$
\hat{s}_{1}\left(t-\tau_{1}\right)=\hat{\alpha}_{1} \sqrt{2 P} \bar{b}_{1}\left(t-\tau_{1}\right) c_{1}\left(t-\tau_{1}\right) \cos \left(\omega_{c} t+\hat{\phi}_{1}\right) .
$$

Therefore, at the input to the second serial stage MF receiver we subtract $\hat{s}_{1}\left(t-\tau_{1}\right)$ from the composite signal $r(t)$, yielding

$$
\begin{aligned}
& r_{s, 2}(t) \\
& =r(t)-\hat{s}_{1}\left(t-\tau_{1}\right) \\
& =n(t)+\sum_{k=2}^{K} \alpha_{k} \sqrt{2 P} c_{k}\left(t-\tau_{k}\right) b_{k}\left(t-\tau_{k}\right) \cos \left(\omega_{c} t+\phi_{k}\right) \\
& \quad+\alpha_{1} \sqrt{2 P} b_{1}\left(t-\tau_{1}\right) c_{1}\left(t-\tau_{1}\right) \cos \left(\omega_{c} t+\phi_{1}\right) \\
& \quad-\hat{\alpha}_{1} \sqrt{2 P} \bar{b}_{1}\left(t-\tau_{1}\right) c_{1}\left(t-\tau_{1}\right) \cos \left(\omega_{c} t+\hat{\phi}_{1}\right)
\end{aligned}
$$

where $\hat{\alpha}_{1}=\alpha_{1}+\triangle \alpha_{1}$ and $\hat{\phi}_{1}=\phi_{1}+\triangle \phi_{1}$. At the output of second stage receiver the decision statistic is

$$
\begin{aligned}
Z_{s, 2} & =\int_{0}^{T} r_{s, 2}(t) c_{2}(t) \cos \left(\omega_{c} t+\hat{\phi}_{2}\right) d t \\
& =D_{s, 2}+I_{s, 2}+E_{s, 2}+\eta
\end{aligned}
$$

where the new term $E_{s, 2}$ is the residual MAI arising from the nonperfect cancellation of the strongest signal at the input to the second stage. This residual MAI is due to both the channel estimation and detection errors in the preceeding MF receiver. Similarly, the expression for each term in (26) are

$$
\begin{aligned}
D_{s, 2}= & \alpha_{2} T \sqrt{\frac{P}{2}} b_{2}(t) \cos \left(\triangle \phi_{2}\right) \\
I_{s, 2}= & \sum_{k=3}^{K} \alpha_{k} \sqrt{\frac{P}{2}} \int_{0}^{T} b_{k}\left(t-\tau_{k}\right) c_{k}\left(t-\tau_{k}\right) \\
& \cdot c_{2}(t) \cos \left(\phi_{k}-\hat{\phi}_{2}\right) d t \\
\eta= & \int_{0}^{T} n(t) c_{2}(t) \cos \left(\omega_{c} t+\hat{\phi}_{2}\right) d t
\end{aligned}
$$

and

$$
\begin{aligned}
& E_{s, 2} \\
& =\alpha_{1} \sqrt{\frac{P}{2}} \int_{0}^{T} b_{1}\left(t-\tau_{1}\right) c_{1}\left(t-\tau_{1}\right) c_{2}(t) \cos \left(\phi_{1}-\hat{\phi}_{2}\right) d t \\
& \quad-\left(\alpha_{1}+\triangle \alpha_{1}\right) \sqrt{\frac{P}{2}} \int_{0}^{T} \\
& \quad \cdot \bar{b}_{1}\left(t-\tau_{1}\right) c_{1}\left(t-\tau_{1}\right) c_{2}(t) \cos \left(\hat{\phi}_{1}-\hat{\phi}_{2}\right) d t \\
& \approx \\
& \alpha_{1} \sqrt{\frac{P}{2}} \int_{0}^{T} \breve{b}_{1}\left(t-\tau_{1}\right) c_{1}\left(t-\tau_{1}\right) c_{2}(t) \cos \left(\phi_{1}-\hat{\phi}_{2}\right) d t \\
& \quad+\Delta \phi_{1} \sqrt{\frac{P}{2}} \int_{0}^{T} \bar{b}_{1}\left(t-\tau_{1}\right) c_{1}\left(t-\tau_{1}\right) c_{2}(t) \sin \left(\phi_{1}-\hat{\phi}_{2}\right) d t \\
& \quad-\Delta \alpha_{1} \sqrt{\frac{P}{2}} \int_{0}^{T} \bar{b}_{1}\left(t-\tau_{1}\right) c_{1}\left(t-\tau_{1}\right) c_{2}(t) \cos \left(\hat{\phi}_{1}-\hat{\phi}_{2}\right) d t
\end{aligned}
$$

where $\stackrel{\vee}{b}(t)=b_{1}(t)-\bar{b}_{1}(t)$. The detail derivation of $E_{s, 2}$ is given in Section $\mathrm{C}$ of the Appendix. The variance of $\eta$ is given 
by (18) and the conditional variance of $I_{s, 2}$ and the variance of $E_{s, 2}$ are as follows:

$$
\begin{aligned}
\operatorname{Var}^{\prime}\left(I_{s, 2}\right)= & \frac{N T_{c}^{2}}{3} P \sum_{k=3}^{K} \alpha_{k}^{2} E\left[\cos ^{2}\left(\phi_{k}-\hat{\phi}_{2}\right)\right] \\
= & \frac{T^{2}}{6 N} P \sum_{k=3}^{K} \alpha_{k}^{2} \\
\operatorname{Var}\left(E_{s, 2}\right)= & E\left[\alpha_{1}^{2}\right]\left(4 P b_{s, 1}\right) \frac{T^{2}}{6 N} P+\sigma_{\triangle \phi_{1}}^{2} \frac{T^{2}}{6 N} P \\
& +\sigma_{\triangle \alpha_{1}}^{2} \frac{T^{2}}{6 N} P
\end{aligned}
$$

as $E\left[\cos \left(\phi_{k}-\hat{\phi}_{2}\right)\right]=0$ and $E\left[\cos ^{2}\left(\phi_{k}-\hat{\phi}_{2}\right)\right]=\frac{1}{2}$. For a detailed derivation of the previous expressions, see (64) and Section D of the Appendix, respectively. Therefore, the conditional SIR at the output of the second receiver is

$$
\operatorname{SIR}_{s, 2}^{\prime}=\frac{D_{s, 2}^{2}}{\operatorname{Var}^{\prime}\left(I_{s, 2}\right)+\operatorname{Var}\left(E_{s, 2}\right)+\operatorname{Var}(\eta)}
$$

where

$$
D_{s, 2}^{2}=\alpha_{2}^{2} T^{2} \frac{P}{2}\left(\cos ^{2}\left(\triangle \phi_{2}\right)\right) .
$$

When $\sigma_{\triangle \phi_{1}}^{2}=\sigma_{\triangle \alpha_{1}}^{2}=0$, (33) reverts to our original (19) for the conditional SIR with perfect channel estimation, but with the index 1 becoming 2 . At the input of the third serial stage receiver, we have

$$
\begin{aligned}
r_{s, 3}(t)= & r_{s, 2}(t)-\hat{s}_{2}\left(t-\tau_{2}\right) \\
= & n(t)+\sum_{k=3}^{K} \alpha_{k} \sqrt{2 P} c_{k}\left(t-\tau_{k}\right) b_{k}\left(t-\tau_{k}\right) \cos \left(\omega_{c} t+\phi_{k}\right) \\
& +\alpha_{1} \sqrt{2 P} c_{1}\left(t-\tau_{1}\right) b_{1}\left(t-\tau_{1}\right) \cos \left(\omega_{c} t+\phi_{1}\right) \\
& -\hat{\alpha}_{1} \sqrt{2 P} c_{1}\left(t-\tau_{1}\right) \bar{b}_{1}\left(t-\tau_{1}\right) \cos \left(\omega_{c} t+\hat{\phi}_{1}\right) \\
& +\alpha_{2} \sqrt{2 P} c_{2}\left(t-\tau_{2}\right) b_{2}\left(t-\tau_{2}\right) \cos \left(\omega_{c} t+\phi_{2}\right) \\
& -\hat{\alpha}_{2} \sqrt{2 P} c_{2}\left(t-\tau_{2}\right) \bar{b}_{2}\left(t-\tau_{2}\right) \cos \left(\omega_{c} t+\hat{\phi}_{2}\right)
\end{aligned}
$$

The decision statistic at the output of third MF receiver in the SSC stage is

$$
\begin{aligned}
Z_{s, 3} & =\int_{0}^{T} r_{s, 3}(t) c_{3}(t) \cos \left(\omega_{c} t+\hat{\phi}_{3}\right) d t \\
& =D_{s, 3}+I_{s, 3}+E_{s, 3}+\eta
\end{aligned}
$$

where

$$
\begin{aligned}
& D_{s, 3} \\
& =\alpha_{3} T \sqrt{\frac{P}{2}} b_{3}(t) \cos \left(\triangle \phi_{3}\right)
\end{aligned}
$$

$$
\begin{aligned}
& I_{s, 3} \sum_{k=4}^{K} \alpha_{k} \sqrt{\frac{P}{2}} \int_{0}^{T} b_{k}\left(t-\tau_{k}\right) c_{k}\left(t-\tau_{k}\right) c_{3}(t) \cos \left(\phi_{k}-\hat{\phi}_{3}\right) d t \\
& E_{s, 3} \\
& =\alpha_{1} \sqrt{\frac{P}{2}} \int_{0}^{T} b_{1}\left(t-\tau_{1}\right) c_{1}\left(t-\tau_{1}\right) c_{3}(t) \cos \left(\phi_{1}-\hat{\phi}_{3}\right) d t \\
& \quad+\triangle \phi_{1} \sqrt{\frac{P}{2}} \int_{0}^{T} \bar{b}_{1}\left(t-\tau_{1}\right) c_{1}\left(t-\tau_{1}\right) c_{3}(t) \sin \left(\phi_{1}-\hat{\phi}_{3}\right) d t \\
& \quad-\triangle \alpha_{1} \sqrt{\frac{P}{2}} \int_{0}^{T} \bar{b}_{1}\left(t-\tau_{1}\right) c_{1}\left(t-\tau_{1}\right) c_{3}(t) \cos \left(\hat{\phi}_{1}-\hat{\phi}_{3}\right) d t \\
& \quad+\alpha_{2} \sqrt{\frac{P}{2}} \int_{0}^{T} b_{2}\left(t-\tau_{2}\right) c_{2}\left(t-\tau_{2}\right) c_{3}(t) \cos \left(\phi_{2}-\hat{\phi}_{3}\right) d t \\
& \quad+\triangle \phi_{2} \sqrt{\frac{P}{2}} \int_{0}^{T} \bar{b}_{2}\left(t-\tau_{2}\right) c_{2}\left(t-\tau_{2}\right) c_{3}(t) \sin \left(\phi_{2}-\hat{\phi}_{3}\right) d t \\
& \quad-\triangle \alpha_{2} \sqrt{\frac{P}{2}} \int_{0}^{T} \bar{b}_{2}\left(t-\tau_{2}\right) c_{2}\left(t-\tau_{2}\right) c_{3}(t) \cos \left(\hat{\phi}_{2}-\hat{\phi}_{3}\right) d t
\end{aligned}
$$

and $\eta$ is defined in (15). Following the same procedure as in the previous receivers, the conditional SIR for the third receiver output is

$$
\operatorname{SIR}_{s, 3}^{\prime}=\frac{D_{s, 3}^{2}}{\operatorname{Var}^{\prime}\left(I_{s, 3}\right)+\operatorname{Var}\left(E_{s, 3}\right)+\operatorname{Var}(\eta)}
$$

where

$$
\begin{aligned}
D_{s, 3}^{2} & =\alpha_{3}^{2} T^{2} \frac{P}{2}\left(\cos ^{2}\left(\triangle \phi_{3}\right)\right) \\
\operatorname{Var}^{\prime}\left(I_{s, 3}\right) & =\frac{T^{2}}{6 N} P \sum_{k=4}^{K} \alpha_{k}^{2}
\end{aligned}
$$

$$
\begin{aligned}
& \operatorname{Var}\left(E_{s, 3}\right) \\
& =E\left[\alpha_{1}^{2}\right]\left(4 P b_{s, 1}\right) \frac{T^{2}}{6 N} P+\sigma_{\triangle \phi_{1}}^{2} \frac{T^{2}}{6 N} P+\sigma_{\triangle \alpha_{1}}^{2} \frac{T^{2}}{6 N} P \\
& \quad+E\left[\alpha_{2}^{2}\right]\left(4 P b_{s, 2}\right) \frac{T^{2}}{6 N} P+\sigma_{\triangle \phi_{2}}^{2} \frac{T^{2}}{6 N} P+\sigma_{\triangle \alpha_{2}}^{2} \frac{T^{2}}{6 N} P
\end{aligned}
$$

and the $\operatorname{Var}(\eta)$ is as before. Based on mathematical induction, we can show that the conditional SIR at the output of the $k$ th serial stage MF receiver is (46), given at the bottom of the page, where $E_{b}$ is the energy per bit and $N_{o}$ is the single-sided PSD of the AWGN process. Now the SIR is conditioned on both $\Delta \phi_{k}$ and $\alpha_{j}$ as well as on the ranked Rayleigh amplitude, $\alpha_{k}$. To remove this conditionality, we first perform the expectation operation on the conditional SIR with respect to the phase error term, $\triangle \phi_{k}$, and the $j$ th-ranked signal amplitude $\alpha_{j}$, followed by averaging the conditional probability of bit error with the probability density function of the $k$ th-ranked signal amplitude over

$$
\operatorname{SIR}_{s, k}^{\prime}=\frac{\alpha_{k}^{2} T^{2} \frac{P}{2} \cos ^{2}\left(\triangle \phi_{k}\right)}{\frac{N_{o} T}{4}+\frac{T^{2}}{6 N} P \sum_{j=k+1}^{K} \alpha_{j}^{2}+\frac{T^{2}}{6 N} P \sum_{v=1}^{k-1} E\left[\alpha_{v}^{2}\right]\left(4 P b_{s, v}\right)+\frac{T^{2}}{6 N} P\left(\sigma_{\triangle \alpha}^{2}+\sigma_{\triangle \phi}^{2}\right)(k-1)}
$$


the range of all possible amplitude values. We therefore have (47), given at the bottom of the page, since from [15] we know that $E[\cos (b x)]=\exp \left(-b^{2} \frac{\sigma_{x}^{2}}{2}\right)$, where $b$ is a constant and $\sigma_{x}^{2}$ is the variance of the Gaussian random variable $x$. Therefore the unconditional $k$ th stage probability of bit error is

$$
\begin{aligned}
P b_{s, k} & =E\left[Q\left(\sqrt{E\left[\mathrm{SIR}_{s, k}^{\prime}\right]}\right)\right] \\
& =\int_{0}^{\infty} Q\left(\sqrt{E\left[\mathrm{SIR}_{s, k}^{\prime}\right]}\right) f_{k, K}\left(\alpha_{k}\right) d \alpha_{k}
\end{aligned}
$$

where $f_{k, K}\left(\alpha_{k}\right)$ is the PDF of the $k$ th-ranked Rayleigh signal amplitude [16] and has the following expression:

$$
f_{k, K}\left(\alpha_{k}\right)=\frac{K !}{(K-k) !(k-1) !} F(\alpha)^{K-k}(1-F(\alpha))^{k-1} f(\alpha)
$$

where $f(\alpha)$ and $F(\alpha)$ are the PDF and CDF of the Rayleigh distribution of the flat fading signal amplitudes having a mean square value $\beta$ of one and is given by

$$
\begin{aligned}
f(\alpha) & =\frac{2 \alpha}{\beta} \exp \left(\frac{-\alpha^{2}}{\beta}\right) \\
& =2 \alpha \exp \left(-\alpha^{2}\right) \\
F(\alpha) & =1-\exp \left(-\alpha^{2}\right)
\end{aligned}
$$

with

$$
E\left[\alpha_{j}^{2}\right]=\int_{0}^{\infty} \alpha_{j}^{2} f_{j, K}\left(\alpha_{j}\right) d\left(\alpha_{j}\right)
$$

The mean square value of one is chosen to allow for ease of simulation where the average power of each user's Rayleigh fading statistic is normalized to the value of unity. Equation (48) of $P b_{s, k} ; k=1,2, \ldots K$ provides us with an expression for the probability of bit error at the output of each serial stage receiver in the SSC stage. The average probability of bit error for the SSC stage is then obtained by performing an arithmetic averaging of the sum of the individual probability of bit error at the output of each serial stage receiver.

\section{SSPC Scheme Model}

In order to formulate the complete analytical model for the SSPC receiver, we first need to model the impact of detection errors in the previous serial stage on the current MF output of the parallel stage. Observe that, for the SSPC receiver, the impact of detection errors on the first parallel cancellation stage (SPC) outputs is due to the outputs of the SSC stage. To obtain the complete analytical model, we follow a similar approach to the one used in the SSC stage. We first obtain the SIR at the output of each MF receiver in the first parallel cancellation stage. With these SIR's we can then obtain the individual probability of bit error. This process is repeated for the second parallel cancellation stage, and so on. The average probability of bit error for a particular parallel stage is obtained as for the SSC stage.

Therefore, the input to the MF for the strongest MS's signal at the first parallel cancellation stage, denoted by the subscript $p 1$, is

$$
\begin{aligned}
r_{p 1,1}= & r(t)-\sum_{k=2}^{K} \hat{s}_{k}\left(t-\tau_{k}\right) \\
= & n(t)+\alpha_{1} \sqrt{2 P} c_{1}\left(t-\tau_{1}\right) b_{1}\left(t-\tau_{1}\right) \cos \left(\omega_{c} t+\phi_{1}\right) \\
& +\sum_{k=2}^{K}\left[s_{k}\left(t-\tau_{k}\right)-\hat{s}_{k}\left(t-\tau_{k}\right)\right] \\
= & n(t)+\alpha_{1} \sqrt{2 P} c_{1}\left(t-\tau_{1}\right) b_{1}\left(t-\tau_{1}\right) \cos \left(\omega_{c} t+\phi_{1}\right) \\
& +\sum_{k=2}^{K}\left[\alpha_{k} \sqrt{2 P} c_{k}\left(t-\tau_{k}\right) b_{k}\left(t-\tau_{k}\right) \cos \left(\omega_{c} t+\phi_{k}\right)\right. \\
& \left.-\hat{\alpha}_{k} \sqrt{2 P} c_{k}\left(t-\tau_{k}\right) \bar{b}_{k}\left(t-\tau_{k}\right) \cos \left(\omega_{c} t+\hat{\phi}_{k}\right)\right] .
\end{aligned}
$$

At the output of this receiver the signal at the input to the decision circuit is the decision statistic $Z_{p 1,1}$ given by

$$
\begin{aligned}
Z_{p 1,1} & =\int_{0}^{T} r_{p 1,1}(t) c_{1}(t) \cos \left(\omega_{c} t+\hat{\phi}_{1}\right) d t \\
& =D_{p 1,1}+E_{p 1,1}+\eta
\end{aligned}
$$

where $D_{p 1,1}$ is the desired signal of the strongest user (i.e., $k=$ 1 ), $E_{p 1,1}$ and $\eta$ are the zero mean Gaussian random variables due to both the channel estimation and detection errors in the SSC stage and AWGN, respectively. The expressions for these components of $Z_{p 1,1}$ are

$$
D_{p 1,1}=\alpha_{1} T \sqrt{\frac{P}{2}} \cos \left(\triangle \phi_{1}\right)
$$

and (56), given at the bottom of the next page, where (56) is derived on a similar assumption as (30) with $b_{1}(t)=b_{1}(t)-$ $\bar{b}_{1}(t)$, and the AWGN component is given by

$$
\eta=\int_{0}^{T} n(t) c_{1}(t) \cos \left(\omega_{c} t+\hat{\phi}_{1}\right) d t
$$

The variance of $E_{p 1,1}$ can be shown to be

$$
\operatorname{Var}\left(E_{p 1,1}\right)=\sum_{k=2}^{K} \frac{T^{2}}{6 N} P\left(E\left[\alpha_{k}^{2}\right]\left(4 P b_{s, k}\right)+\sigma_{\triangle \phi_{k}}^{2}+\sigma_{\triangle \alpha_{k}}^{2}\right)
$$

$$
\begin{aligned}
E\left[\operatorname{SIR}_{s, k}^{\prime}\right] & =\left\{\frac{\alpha_{k}^{2} T^{2} \frac{P}{2} E\left[\cos ^{2}\left(\triangle \phi_{k}\right)\right]}{\frac{N_{o} T}{4}+\frac{T^{2}}{6 N} P \sum_{j=k+1}^{K} E\left[\alpha_{j}^{2}\right]+\frac{T^{2}}{6 N} P \sum_{v=1}^{k-1} E\left[\alpha_{v}^{2}\right]\left(4 P b_{s, v}\right)+\frac{T^{2}}{6 N} P\left(\sigma_{\triangle \alpha}^{2}+\sigma_{\triangle \phi}^{2}\right)(k-1)}\right\} \\
& =\left\{\frac{\alpha_{k}^{2} \frac{1}{2}\left[1+\exp \left(-2 \sigma_{\triangle \phi_{k}}^{2}\right)\right]}{\frac{N_{o}}{2 E_{b}}+\frac{1}{3 N} \sum_{j=k+1}^{K} E\left[\alpha_{j}^{2}\right]+\frac{1}{3 N} \sum_{v=1}^{k-1} E\left[\alpha_{v}^{2}\right]\left(4 P b_{s, v}\right)+\frac{1}{3 N}\left(\sigma_{\triangle \alpha}^{2}+\sigma_{\triangle \phi}^{2}\right)(k-1)}\right\}
\end{aligned}
$$


where $E\left[\alpha_{k}^{2}\right]$ is defined by (52). The variance expression in (58) is obtained in a similar way to that in (32). Equation (58) accounts for the impact of detection errors in the SSC stage on the output of the individual MF's at the first parallel stage. The conditional SIR at the output of the MF for the strongest MS's signal is therefore

$$
\operatorname{SIR}_{p 1,1}^{\prime}=\frac{D_{p 1,1}^{2}}{\operatorname{Var}\left(E_{p 1,1}\right)+\operatorname{Var}(\eta)} .
$$

Having obtained the conditional SIR, we can now proceed to obtain the unconditional average probability of bit error as

$$
\begin{aligned}
P b_{p 1,1} & =E\left[Q\left(\sqrt{E\left[\operatorname{SIR}_{p 1,1}^{\prime}\right]}\right)\right] \\
& =\int_{0}^{\infty} Q\left(\sqrt{E\left[\operatorname{SIR}_{p 1,1}^{\prime}\right]}\right) f_{1, K}\left(\alpha_{1}\right) d \alpha_{1}
\end{aligned}
$$

where $f_{1, K}$ is defined in (49). The above process is repeated to obtain the conditional $(K-1)$ SIR's at the outputs of the first parallel stage. The individual average probability of bit error, $P b_{p 1, k} ; k=1,2, \ldots K$, thus obtained is then summed and averaged over all the $K$ users to give the average probability of bit error at the output of first parallel cancellation stage.

To obtain the average probability of bit error for the next successive parallel stage, we proceed as before. Assuming that we deploy up to $V ; v=1,2, \ldots V$, SPC's, the residual MAI variance for the $j$ th MS at the output of $v$ th SPC is

$$
\begin{aligned}
& \operatorname{Var}\left(E_{p v, j}\right) \\
& =\frac{T^{2}}{6 N} P\left[\sum_{k=1, k \neq j}^{K}\left(E\left[\alpha_{k}^{2}\right]\left(4 P b_{p(v-1), k}\right)+\sigma_{\triangle \phi_{k}}^{2}+\sigma_{\triangle \alpha_{k}}^{2}\right)\right] .
\end{aligned}
$$

Once the variance of $E_{p v, j}$ for the $j$ th MS is obtained we can proceed to obtain the probability of bit error $P b_{p v, j}$ as before.

\section{ANALYTICAL AND SIMULATION RESUlTS}

This section presents the results obtained in the simulation of the SSPC scheme operating in the presence of flat Rayleigh fading channels.

The processing gain was $N=127$. The Rayleigh fading statistic was generated for a Doppler bandwidth of $100 \mathrm{~Hz}$ and

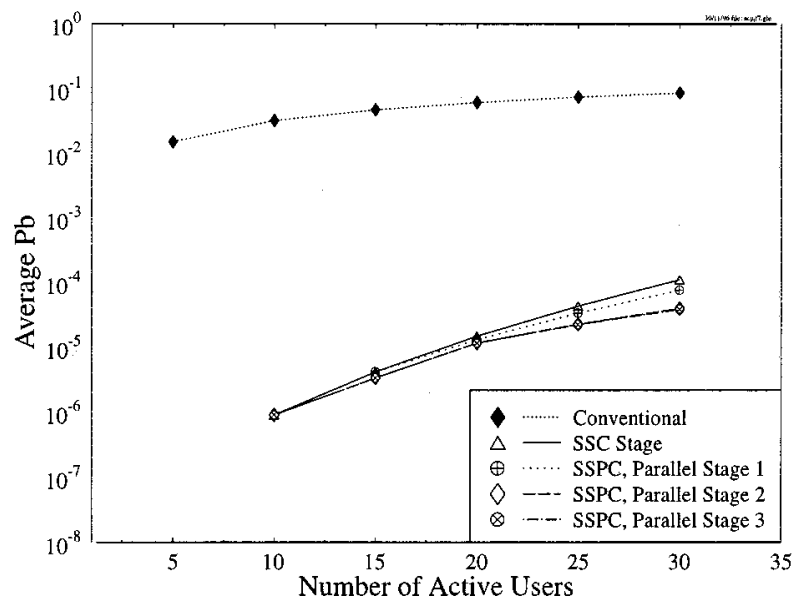

Fig. 7. Simulation performance of the SSPC scheme versus conventional detection scheme without AWGN, but with perfect ranking and perfect channel estimation and $N=127$.

individual user data transmission rate was $8 \mathrm{kbps}$. Two simulation models were adopted in order to curtail the simulation time. The first was a chip and phase synchronous simulation (CSPS), whereas the second technique was chip synchronous, but phase asynchronous (CSPA). The first technique was employed for perfect channel estimation simulations while the second technique was employed for simulating the impact of phase channel estimation errors. The CSPS and CSPA conditions were adopted to alleviate the complexity of the simulation. As such, the analytical results shown in this section were obtained using the corresponding CSPS and CSPA versions of the SIR expressions.

The channel estimation errors were generated independently for each stage in both the serial and parallel stages according to the required variances.

\section{A. Performance Comparison with MSPC Scheme}

1) Impact of Perfect Channel Estimation (PCE): The performance of both successive serial cancellation stage (SSC) and combined successive serial parallel cancellation stages (SSPC) for transmissions over flat fading channels are shown in Fig. 7 for $5 \leq K \leq 30$. Also displayed is the performance of

$$
\begin{aligned}
E_{p 1,2}=\sum_{k=2}^{K}\left[\alpha_{k} \sqrt{\frac{P}{2}} \int_{0}^{T} b_{k}\left(t-\tau_{k}\right) c_{k}\left(t-\tau_{k}\right) c_{1}(t) \cos \left(\phi_{k}-\hat{\phi}_{1}\right) d t\right. \\
\left.\quad-\left(\alpha_{k}+\triangle \alpha_{k}\right) \sqrt{\frac{P}{2}} \int_{0}^{T} \bar{b}_{k}\left(t-\tau_{k}\right) c_{k}\left(t-\tau_{k}\right) c_{1}(t) \cos \left(\hat{\phi}_{k}-\hat{\phi}_{1}\right) d t\right] \\
=\sum_{k=2}^{K}\left[\alpha_{k} \sqrt{\frac{P}{2}} \int_{0}^{T} b_{k}\left(t-\tau_{k}\right) c_{k}\left(t-\tau_{1}\right) c_{1}(t) \cos \left(\phi_{k}-\hat{\phi}_{1}\right) d t\right. \\
\left.+\triangle \phi_{k} \sqrt{\frac{P}{2}} \int_{0}^{T} \bar{b}_{k}\left(t-\tau_{k}\right) c_{k}\left(t-\tau_{k}\right) c_{1}(t) \sin \left(\phi_{k}-\hat{\phi}_{1}\right) d t\right] \\
\left.-\triangle \alpha_{k} \sqrt{\frac{P}{2}} \int_{0}^{T} \bar{b}_{k}\left(t-\tau_{k}\right) c_{k}\left(t-\tau_{k}\right) c_{1}(t) \cos \left(\hat{\phi}_{k}-\hat{\phi}_{1}\right) d t\right]
\end{aligned}
$$




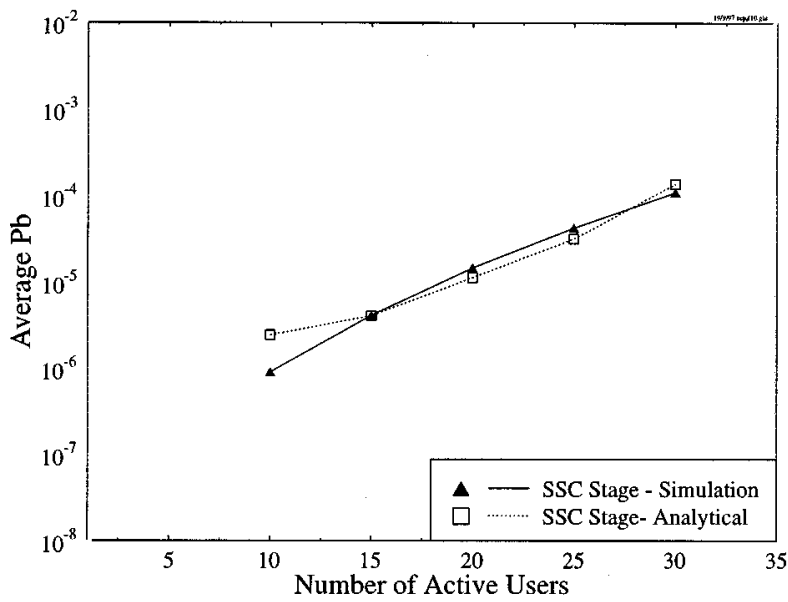

Fig. 8. Analytical versus simulated average probability of bit error for the SSC stage without AWGN, but with perfect ranking, perfect channel estimation, and $N=127$.

the conventional CDMA system that does not use cancellation methods and is employed as a bench marker. The curves are for perfect ranking with the sliding window block ranker, perfect channel estimation, and no receiver noise. The most striking feature of Fig. 7 is that the SSPC scheme outperforms the conventional detection by several orders of magnitude. This was chiefly due to the cancellation of the MAI in the SSPC scheme. Observe that no error was recorded in the simulation for $K=5$. However, there was only a marginal improvement in the average probability of bit error when the successive parallel cancellation stages were added to the successive serial cancellation stage. Only at higher user load (i.e., $K \geq 30$ ) was there an improvement in the bit error probability due to the use of parallel stages. This was due to the individual characteristics of the serial and parallel cancellation schemes. For a smaller number of users, the spread of the Rayleigh-distributed signal amplitudes is wider, and as the successive serial cancellation scheme performs better with unequal powers, it offers the lowest average probability of bit error. Therefore, adding parallel stages does not result in any significant improvement. On the other hand, as the number of users becomes large, the spread of the Rayleigh-distributed amplitudes between each user's signal shrinks and the advantage that accrues from ranking is minimized as the next strongest signal begins to have a significant impact on the detection of the strongest signal. In other words, the effect of the MAI begins to affect the detection. Therefore, in such conditions, parallel stages are able to remove the effect of MAI resulting in a performance improvement. The amount of improvement depends on the size of user base. This is clearly shown in Figs. 9-11.

Fig. 8 depicts the simulation and the analytical results for the successive serial cancellation stage with perfect channel estimation. Notice that for practical user base size, there is close agreement between analytical and simulation results. The inaccuracy of the analytical results when the number of users were less than 15 is principally due to the inaccuracy of the Gaussian approximation technique for small number of users.

Figs. 9-11 shows the analytical results of the average probability of bit error of the SSPC scheme against the mean SNR per

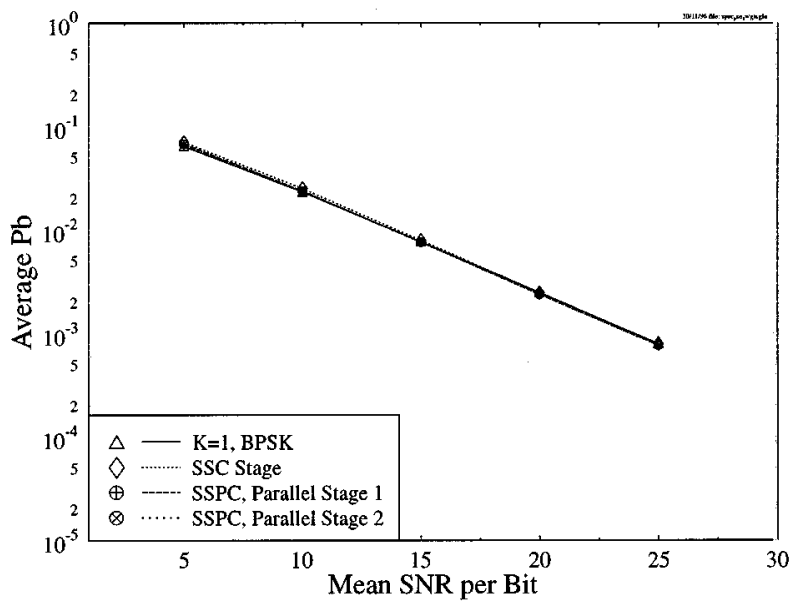

Fig. 9. Analytical performance of the SSPC scheme for $K=20$ users with AWGN, perfect ranking, perfect channel estimation, and $N=127$.

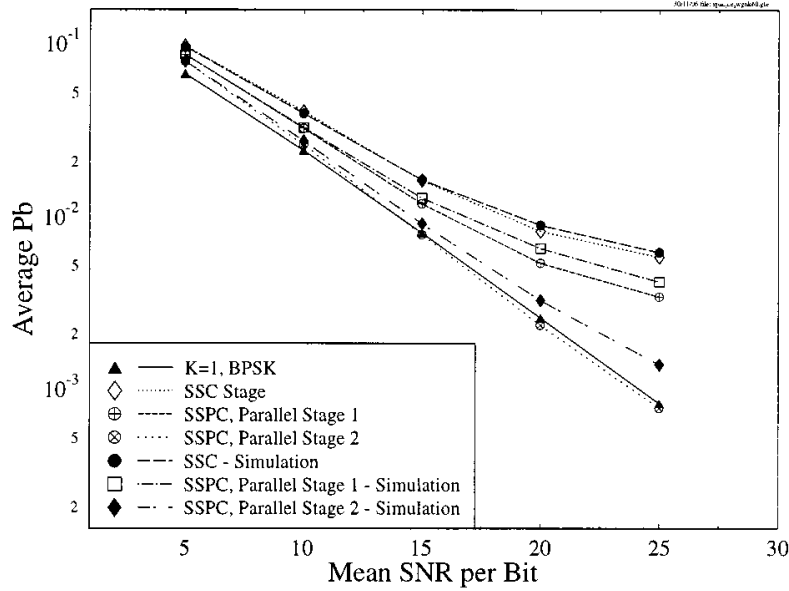

Fig. 10. Analytical and simulated performances of the SSPC scheme for $K=$ 60 users with AWGN, perfect ranking, perfect channel estimation, and $N=$ 127.

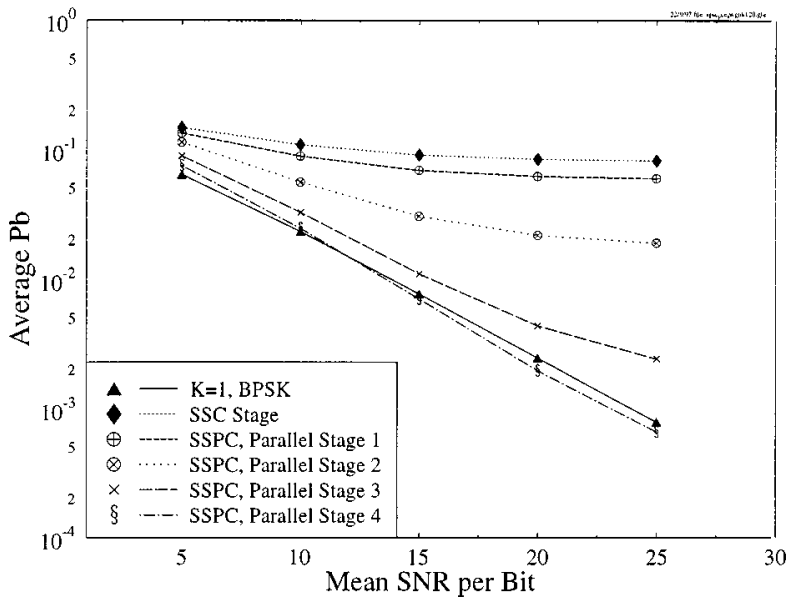

Fig. 11. Analytical performance of the SSPC scheme for $K=120$ users with AWGN, perfect ranking, perfect channel estimation, and $N=127$.

bit for different number of active users $K$. Fig. 10 also includes simulation results, and in each figure the curve for a single user, $K=1$, is displayed. For small numbers of users, $K \leq 20$, 


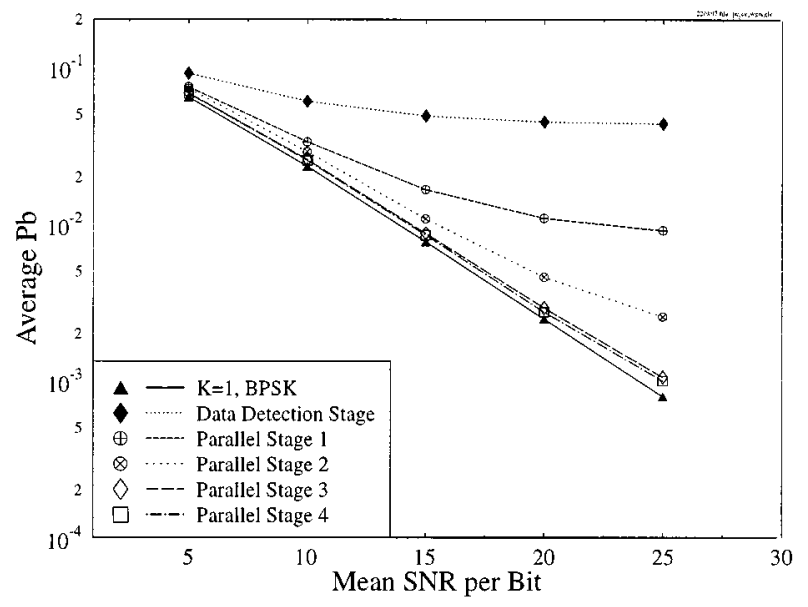

Fig. 12. Analytical performance of the MSPC scheme for $K=20$ users with AWGN and with perfect channel estimation and $N=127$.

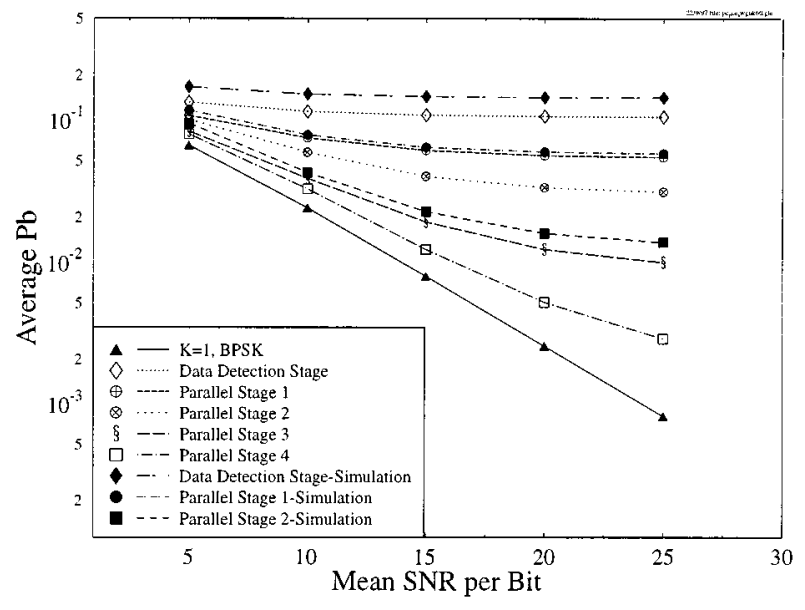

Fig. 13. Analytical and simulated performances of the MSPC scheme for $K=$ 60 users with AWGN and with perfect channel estimation and $N=127$.

the single-user bound is attained at the output of the SSC stage. As such, there is no performance gain in utilizing the SPC's. When the number of users increases, the performance of the serial stage begin to degrade, and an error floor of close to $5 \times 10^{-2}$ occurs for $K=120$. What is significant, is that the single-user performance, $K=1$, can be almost achieved with a further three SPC stages for $K=60$. For $K=120$, the single-user bound was achieved when four parallel cancellation stages were deployed.

The average probability of bit error versus the mean SNR per bit for different number of users for the stand alone MSPC scheme is depicted in Figs. 12-14 for $K=20,60$ and 120, respectively. For small numbers of users (i.e., $K \leq 20$ ), the MSPC scheme performance approaches that of the SSPC scheme by attaining the single-user bound at the third parallel cancellation stage. For $K=60$, the simulation and analytical results are close to each other for the data detection and first parallel cancellation stages. On the other hand, there are some discrepancies between the simulation and analytical results for the second parallel cancellation stage. Nevertheless, as the number of users increases, the the performance of the MSPC scheme degrades

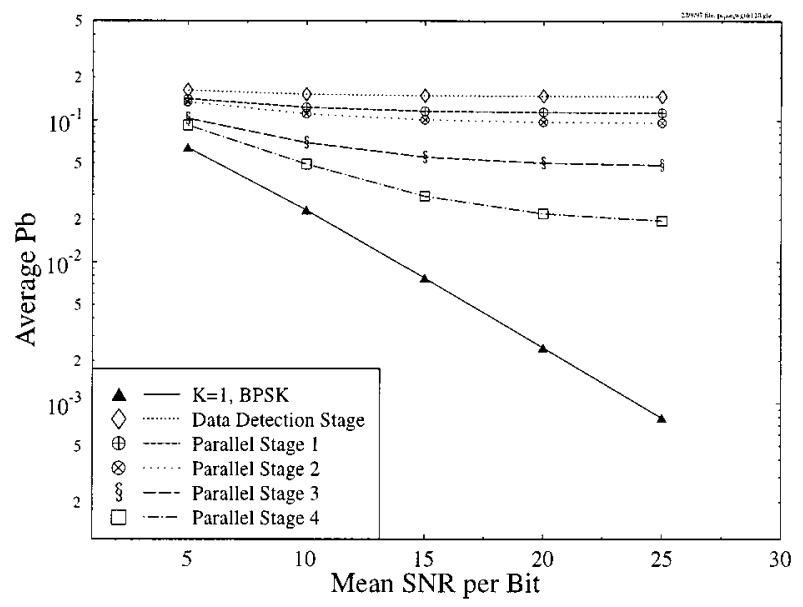

Fig. 14. Analytical performance of the MSPC scheme for $K=120$ users with AWGN and with perfect channel estimation and $N=127$.

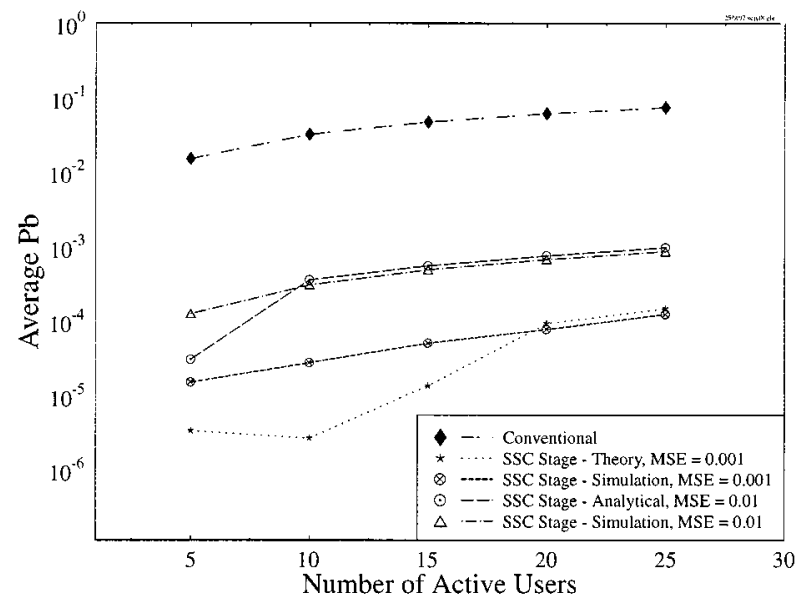

Fig. 15. Performance of the SSPC scheme with amplitude channel estimation errors of MSE $=0.01$ and MSE $=0.001$ and with perfect ranking and $N=127$.

significantly leading to an error floor which further parallel cancellation stages cannot alleviate. This result agrees with those found in [17] and [18]. Observe also that as the number of users increases, the improvement in performance between successive parallel stages begins to shrink.

From the above results, it can be concluded that the SSPC scheme is superior to the MSPC scheme. It also follows that the SSPC scheme can be made adaptive to the offered traffic. For small number of users, the serial stage provides an acceptable performance, and when the number of users exceeds a system threshold the parallel stages can be activated. This adaptive scheme is discussed in the Section V.

2) Impact of Channel Estimation Errors: In this section, we examine the accuracy of the analytical model in the presence of channel estimation errors. Fig. 15 shows the result of SSPC scheme with errors only in the amplitude estimates, while Fig. 16 shows the impact of having both phase and amplitude estimation errors of equal measure. In both figures, the ranking was assumed to be perfect. As expected, the impact of having both types of channel estimation errors was worse than having errors in the amplitude estimates alone. From both figures, it is evident that the analytical results for the serial stage agree 


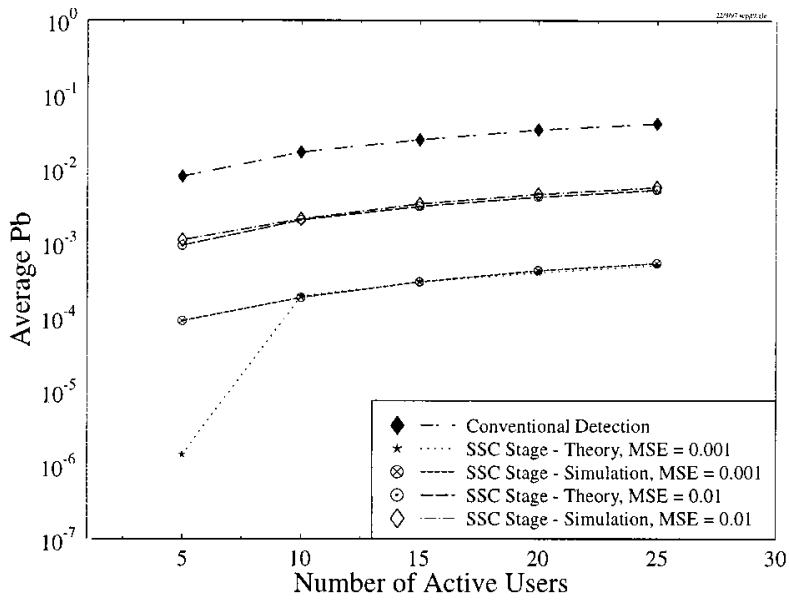

Fig. 16. Performance of the SSPC scheme with both phase and amplitude channel estimation errors of MSE $=0.01$ and MSE $=0.001$ and with perfect ranking and $N=127$

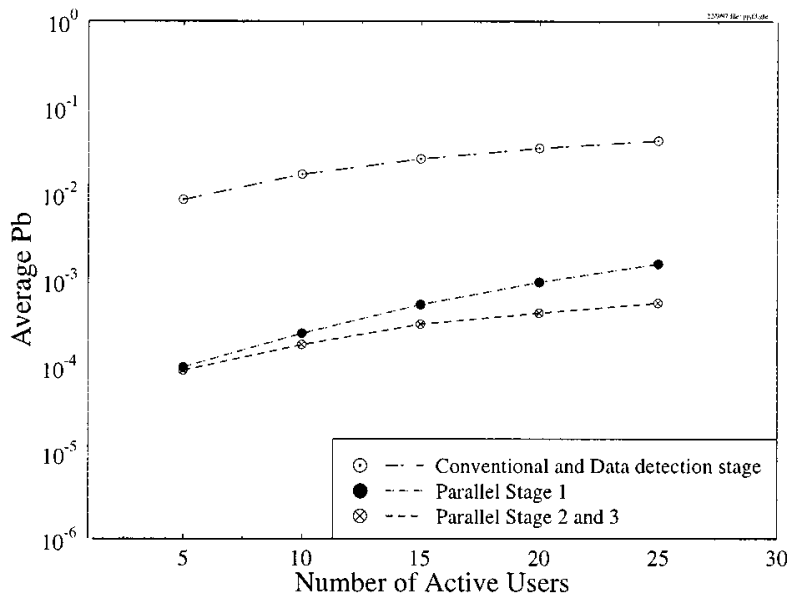

Fig. 17. Simulation performance of the MSPC Scheme with both phase and amplitude channel estimation errors of MSE $=0.001$ and $N=127$.

with the simulations for most practical user base sizes. The optimistic result for the smaller number of users is attributable to the inaccuracy of using the Gaussian approximation technique for small number of users. Applying (7) and (8), the standard deviations $\sigma_{\alpha_{k}}$ and $\sigma_{\phi_{k}}$, were 0.1 and $0.314 \mathrm{rad}$, respectively, for a MSE of 0.01. The corresponding values for a MSE of 0.001 are 0.032 and 0.0993 rad.

In Figs. 15 and 16, only the performances of the serial cancellation stage are shown as there was only a marginal improvement going from serial to parallel stages in the SSPC receiver for small number of users as shown by both analytical and simulation results obtained using the complete SSPC scheme. This was because the dominant source of errors in the serial stage was due to the channel estimation errors and this was further bounded by channel estimation errors in the parallel stages. This second factor is evident from the performance of stand alone MSPC scheme where average probability of bit error curve for the second and third parallel cancellation stages in Fig. 17 is close to the curve obtained from the serial stage having a similar mean square error. This meant that the performance of the stand-

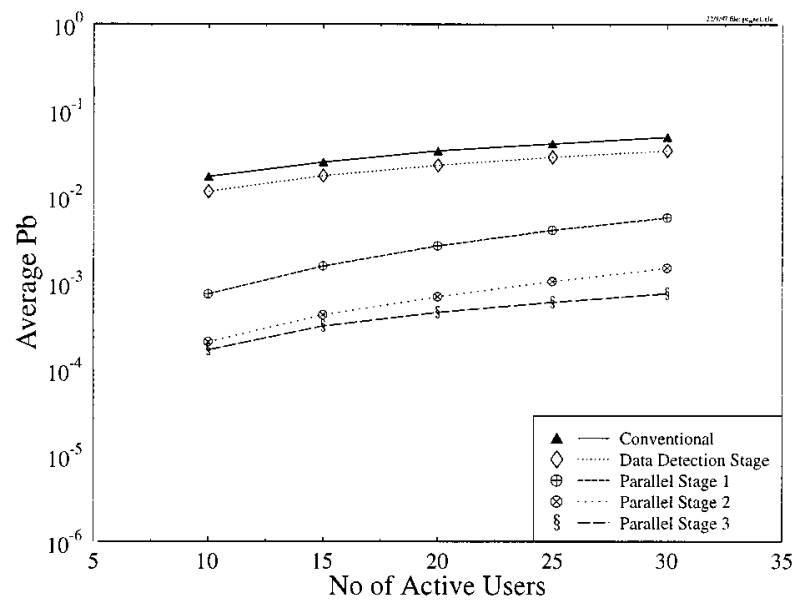

Fig. 18. Analytical performance of the MSPC Scheme with both phase and amplitude channel estimation errors of $\mathrm{MSE}=0.001$ and $N=127$.

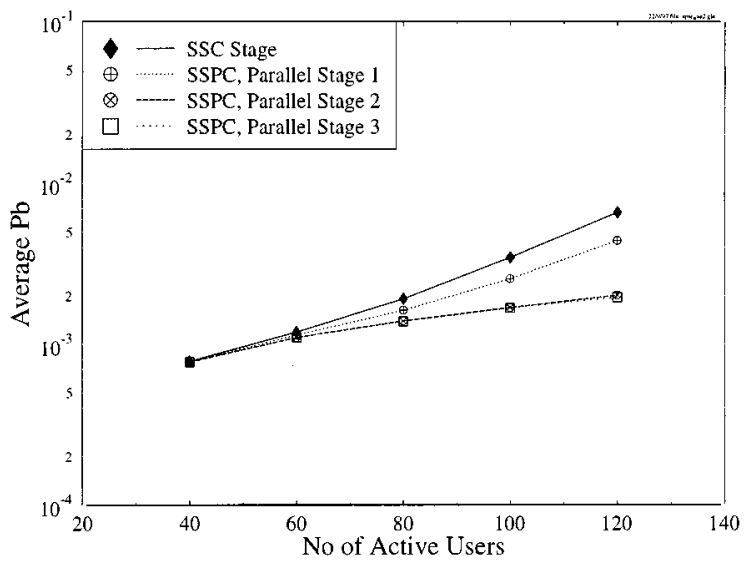

Fig. 19. Analytical performance of the SSPC scheme with both phase and amplitude channel estimation errors of MSE $=0.001$ and for $K=40$ to 120 users and $N=127$

alone cancellation scheme was also bounded by the channel estimation errors. The nonmonotonicity of the analytical results for the case of small channel estimation errors, as depicted in Fig. 15 can be attributed to the inaccuaracy of Gaussian approximation for small number of users. Fig. 18 depicts the corresponding analytical results for the stand-alone parallel cancellation scheme with up to four parallel cancellation stages. Notice that the analytical results obtained are close to the simulation results depicted in Fig. 17 albeit with some discrepancy.

For the SSPC scheme, if the user base was further increased and given a certain small mean square error in the channel estimations, there will be an improvement if parallel stages were deployed. This is shown in Figs. 19 and 20, which depict the performance of the SSPC scheme with channel estimation errors of MSE $=0.001$ and 0.0001 , respectively, for $40 \leq K \leq 120$. For the case of lower channel estimation errors, the improvement obtained from the parallel stages is much more significant. Similarly, Figs. 21 and 22 depict the performance of stand alone MSPC scheme having the same MSE values. As expected, the performance of the stand alone scheme MSPC is much worse for the same mean square channel estimation error. Observe also 


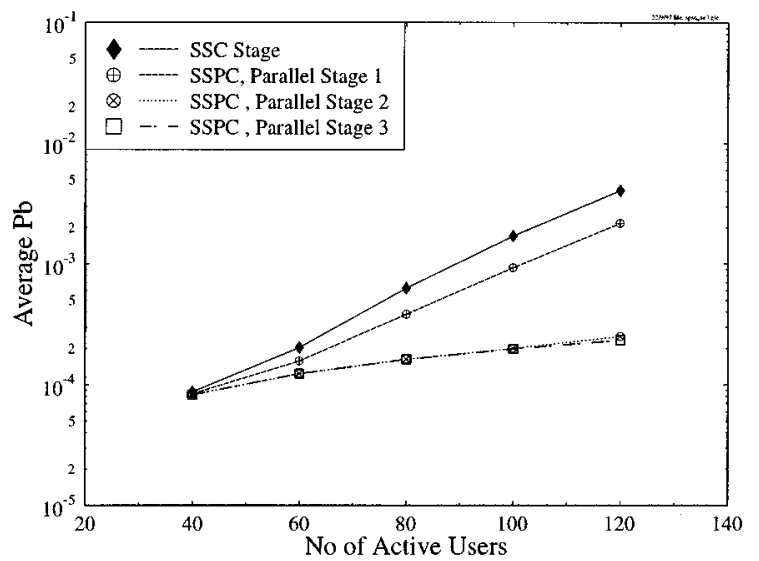

Fig. 20. Analytical performance of the SSPC scheme with both phase and amplitude channel estimation errors of MSE $=0.0001$ and for $K=40$ to 120 users and $N=127$

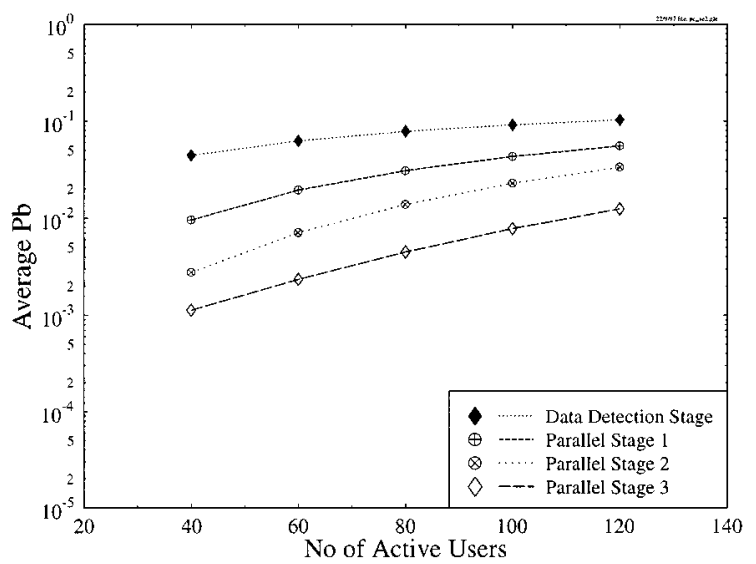

Fig. 21. Analytical performance of the MSPC Scheme with both phase and amplitude channel estimation errors of MSE $=0.001$ and for $K=40$ to 120 users and $N=127$.

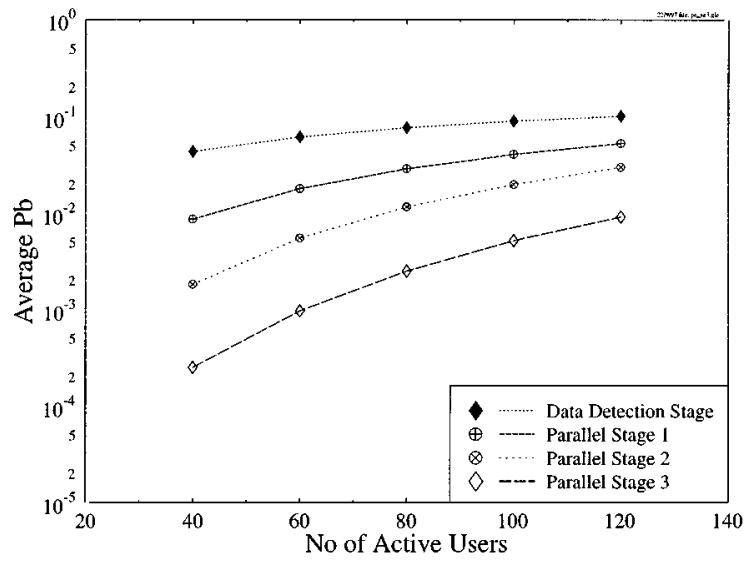

Fig. 22. Analytical performance of the MSPC scheme with both phase and amplitude channel estimation errors of MSE $=0.0001$ and for $K=40$ to 120 users and $N=127$.

that for a large user base, the decrease in channel estimation errors resulted in only a small improvement in the average probability of bit error for the given number of users.

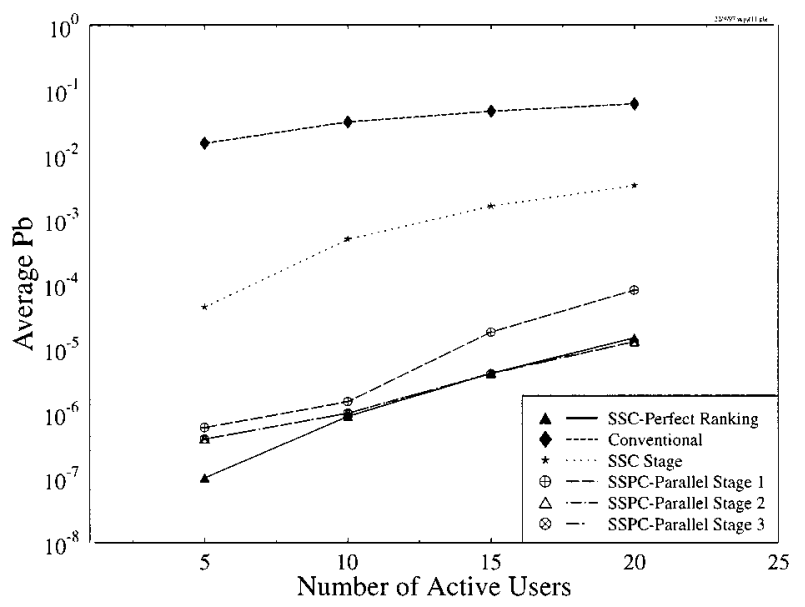

Fig. 23. Impact of imperfect ranking in the SSPC scheme.

\section{B. Impact of Imperfect Ranking}

Fig. 23 depicts the impact of imperfect ranking on the SSPC scheme with perfect channel estimation. Instead of having perfect ranking information for the SSC process, the ranking information used to obtain the results in Fig. 23 was derived from conventional matched filtering. The inaccuracy in ranking led to severe degradation in the performance of the SSC stage when compared to the results obtained with perfect ranking. This is primarily caused by near-far effect of the MAI, resulting from inaccurate ranking, on the demodulation of the desired bit. Notice also that the performance of the SSPC scheme with imperfect ranking was still better than the conventional detection case where single-user MF detection is employed.

The degradation caused by imperfect ranking can however be reduced by the SPC stages in the SSPC receiver to the same level as the result obtained in perfect ranking. If there were channel estimation errors, then based on simulation results there will still be an improvement in the use of additional parallel cancellation stages in the SSPC receiver. However, this improvement is again bounded, as before, by the amount of channel estimation errors.

\section{Impact of Extending Cancellation Frame Size}

The investigation into this subject is motivated by the possibility of improving the average probability of bit error performance of the SSPC scheme by reducing the impact of wrong estimates of the data bit used to regenerate the interference. The rationale behind the use of 3-b cancellation frame size is to ensure that the two partial cross correlations of the interfering users' signals with respect to the desired bit (in this case the center bit in Fig. 2) is removed. To remove the MAI due to these two bits, the two corresponding data bits need to be estimated first. However, the accuracy of this estimation is influenced by other stronger overlapping bits of other users on these two data bits due to the asynchronous nature of the channel. If these stronger interfering bits can be cancelled first, then the accuracy of estimating the two data bits used to regenerate the interference would improve. This procedure necessitates the use of a larger cancellation frame size. The aim of this investigation is to find the cost-effective cancellation frame size for the SSPC scheme. Fig. 24 depicts the simulation results of the average bit error 


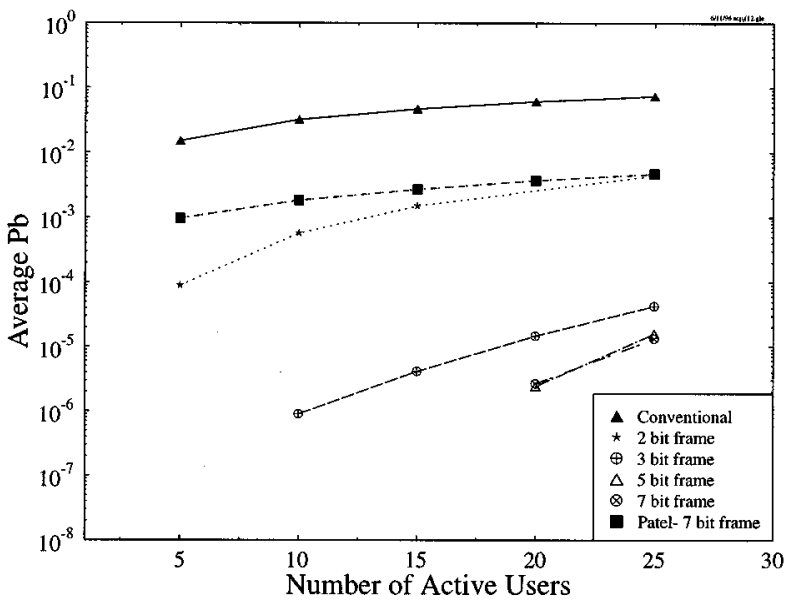

Fig. 24. Impact of the cancellation frame size in the SSPC scheme with perfect ranking.

rate as a function of cancellation frame sizes for the SSC Stage only. The performances of a single-user MF detection scheme and the scheme employed by Patel in [4] for a frame size of $7 \mathrm{~b}$ are also shown for comparison purposes. There was an improvement in enlarging a 2-b frame size to $7 \mathrm{~b}$. The most significant improvement is increasing from the frame size of two to three bits. Observe also that no error was recorded in our simulation when the number of users were between $5-15$. The decrease in average bit error rate was not significant when increasing the frame size from 3 to 5 bits and a negligible improvement for further increase in frame length. This negligible improvement was primarily due to the large direct MAI effect on the desired bit rather than the effect of stronger adjacent overlapping bits alluded to above. From our results we conclude that a 3-b length cancellation frame size is the best compromise in terms of the performance gain and the penalty of increasing the processing delay and signal processing complexity due to the larger frame size [11].

\section{Summary of Our Findings}

For perfect channel estimation and perfect ranking of the received CDMA signal levels and when the number of users are below 25, the SSC stage is able to achieve the single-user bound. This is because the successive cancellation scheme can capitalise on the large differences between each user's received signal power. The probability of the bit error of weaker users is due in part to error propagation from wrong decisions made in regenerating the bits of the stronger users, and from the effect of MAI on the outer two bits the data in the cancellation frame. Increasing the cancellation frame size improves the situation. With perfect channel estimation, the MSPC scheme offers equivalent performance to the SSPC scheme, provided three parallel stages are used for small number of users. Further cancellation stages yields negligible improvement. For user base size approaching the processing gain, the MSPC scheme produces an irreducible error floor.

As the number of users increases the MAI affects the detection performance in the serial stage as errors are induced by the remaining user signals which have signal strengths of a similar order of magnitude. This effect is noticeable for $K \geq 30$, when

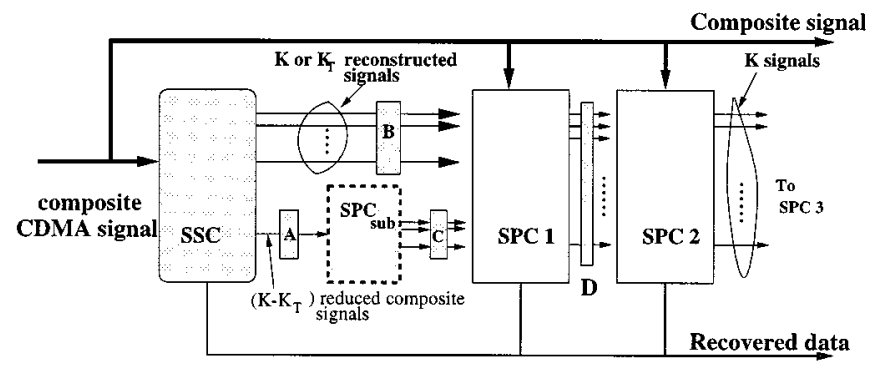

SWITCHES: A,B,C and D

Fig. 25. Adaptive SSPC receiver.

our processing gain is 127 and no FEC coding is used. By the addition of parallel stages in the SSPC receiver, the effect of the MAI on the detection can be gradually reduced if significant number of successive parallel stages are used.

Channel estimation errors increase the detection errors at each serial stage receiver and thereby increase the residual errors. For the stand-alone parallel cancellation scheme, the impact of the channel estimation errors is to increase the error floor as well. The improvement of adding a parallel cancellation stage in the MSPC scheme accrues from partial removal of the MAI in the initial data estimation stage. As the parallel cancellation scheme can only mitigate the effect of MAI, adding parallel cancellation stages to the serial stage in the SSPC scheme does not yield any improvement if the MAI is not the dominant cause of errors.

We have also shown that the 3-b cancellation frame is the best size for successive cancellation. Increasing the cancellation frame size beyond $3 \mathrm{~b}$ yields diminishing returns in performance and an increase in complexity.

\section{AN ADAPTIVE SSPC SCHEME}

Building on the results given in Section IV and summarized in Section D we propose an adaptive SSPC scheme that adapts according to two parameters, namely, the number of users $K$ and the BER. The block diagram of the adaptive SSPC receiver is depicted in Fig. 25. The adaptation algorithm works as follows. If the number of users $K \leq K_{T}$, where $K_{T}$ is a system parameter, only the serial cancellation stage is invoked as there is no advantage in using subsequent parallel cancellation stages, and the delay imposed by the serial cancellation stage is deemed to be acceptable. The recovered data bits are produced by the SSC stage. When the number of users exceed $K_{T}$, then $K_{T}$ users' signals are processed by the SSC and $K-K_{T}$ users' signals are not subjected to the SSC stage processing, but proceed directly to a successive parallel canceller, $\mathrm{SPC}_{\mathrm{sub}}$, via switch A in Fig. 25. After $K_{T}$ users' signals have been subjected to the SSC stage and $K-K_{T}$ users' signals are processed by SPC $_{\text {sub }}$ stage, switches $\mathrm{B}$ and $\mathrm{C}$ are closed and the $K$ CDMA users' signals are processed by the SPC1 stage. If the BER $<\alpha$, a system parameter, the recovered data from SPC1 is delivered to the output for further processing. Should BER $\geq \alpha$, switch D is closed and the CDMA signals from SPC1 are processed by SPC2. SPC stages continue to be employed until BER $\leq \alpha$, or when no significant improvement occurs compared to the previous cancellation stage. 


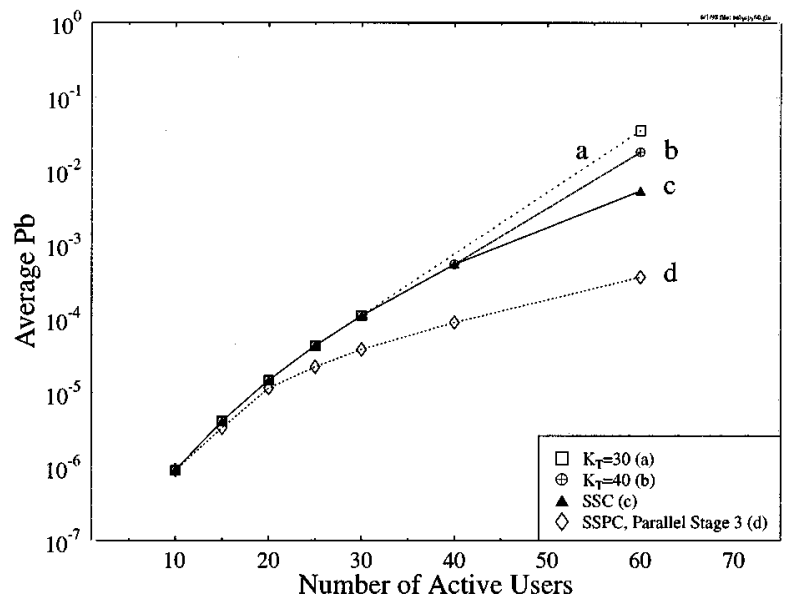

Fig. 26. Simulation results of the adaptive SSPC scheme for different $K_{T}$ values.

In Fig. 26 curve $a$ and curve $b$ show the variations of the average probability of bit error versus the number of active users when SSC in conjunction with the $\mathrm{SPC}_{\text {sub }}$ are used, for $K_{T}=$ 30 and 40 , respectively, with a total of 60 active users. Where three further stages of SPC's are employed, curve $d$ is realized, and this curve is identical to the one using the full serial stage follows by three SPC's. However, employing $K_{T}=30$, the delayed is halved for 60 users. Also shown in Fig. 26 is curve $c$ which depicts the performance of the SSC stage when all the $K$ CDMA signals are processed.

In order to obtain an estimate of the BER for use in the adaptive process, we propose the arrangement depicted in Fig. 27. This scheme is essential as the BER is unknown in actual implementation. Accordingly, for each MF receiver in both the serial and parallel stages, a chip error rate detector is introduced. This detector multiplies the polarity of the recovered data bit $\bar{b}_{k}(t)$ by the received sequence which is then compared on a chip by chip basis with the known spreading sequence for that particular user. The difference in polarity between the actual and recovered chips is logged as a chip errors. The average chip error rate for each SSC and SPC's stages is then obtained by summing all the normalized chip errors and then averaging the sum over the total number of users $K$. Normalization is done over the spreading sequence length and the total number of bits transmitted.

Fig. 28 displays the correlation between the average chip error rates (CER) and the BER for $K=40$ and 60 and with $K_{T}=30$. Using these curves we can obtain an estimate of the BER based on the CER and this is used to compare with $\alpha$, a system parameter, in order to decide whether further SPC stages are required.

\section{CONCLUSION}

We have evaluated the performance of a successive serial parallel cancellation scheme for a CDMA uplink system operating in flat Rayleigh fading channels. The scheme has the potential to reach the single-user bound if sufficiently accurate channel estimates were available for both signal ranking and regeneration. An extension of this scheme to make it adaptive to the number of users is also proposed. This adaptive scheme can reduce the processing delay accrued in the serial successive cancellations stage while still offering the same BER performance at the output of the final parallel cancellation stage.

\section{APPENDIX A}

\section{EXPECTATION OF COSINE FUNCTION WITH Two VARIABLES}

The expectation of $\cos \left(\phi_{k}-\hat{\phi}_{1}\right)$ is

$$
\begin{aligned}
E\left[\cos \left(\phi_{k}-\hat{\phi}_{1}\right)\right] & =E\left[\cos \phi_{k} \cos \hat{\phi}_{1}+\sin \phi_{k} \sin \hat{\phi}_{1}\right] \\
& =0, \quad \text { since } E\left[\sin \phi_{k}\right]=E\left[\cos \phi_{k}\right]=0 .
\end{aligned}
$$

The expectation of $\cos ^{2}\left(\phi_{k}-\hat{\phi}_{1}\right)$ is

$$
\begin{aligned}
E\left[\cos ^{2}\left(\phi_{k}-\hat{\phi}_{1}\right)\right] & \\
= & E\left[\left(\cos \phi_{k} \cos \hat{\phi}_{1}+\sin \phi_{k} \sin \hat{\phi}_{1}\right)^{2}\right] \\
= & E\left[\left(\cos ^{2} \phi_{k} \cos ^{2} \hat{\phi}_{1}+2 \cos \phi_{k} \cos \hat{\phi}_{1} \sin \phi_{k} \sin \hat{\phi}_{1}\right.\right. \\
& \left.\left.+\sin ^{2} \phi_{k} \sin ^{2} \hat{\phi}_{1}\right)\right] \\
= & E\left[\left(\cos ^{2} \phi_{k} \cos ^{2} \hat{\phi}_{1}\right)\right]+E\left[\left(\sin ^{2} \phi_{k} \sin ^{2} \hat{\phi}_{1}\right)\right] \\
= & \frac{1}{2} E\left[\cos ^{2} \hat{\phi}_{1}\right]+\frac{1}{2} E\left[\sin ^{2} \hat{\phi}_{1}\right] \\
= & \frac{1}{2}
\end{aligned}
$$

as $E\left[\cos \phi_{k}\right]=E\left[\sin \phi_{k}\right]=0$, and $E\left[\cos ^{2} \hat{\phi}_{1}\right]=E\left[\sin ^{2} \hat{\phi}_{1}\right]=$ $\frac{1}{2}$ (see Appendix B for derivation details).

\section{APPENDIX B}

EXPECTATION OF SQUARE OF COSINE AND SINE FUNCTIONS

The expectation of $\cos ^{2} \hat{\phi}_{1}$ and $\sin ^{2} \hat{\phi}_{1}$ are

$$
\begin{aligned}
E\left[\cos ^{2} \hat{\phi}_{1}\right] & =E\left[\cos ^{2}\left(\phi_{1}+\triangle \phi_{1}\right)\right] \\
& =E\left[\frac{1}{2}\left(1+\cos \left(2 \phi_{1}+2 \triangle \phi_{1}\right)\right]\right. \\
& =\frac{1}{2}+E\left[\cos 2 \phi_{1} \cos 2 \triangle \phi_{1}-\sin 2 \phi_{1} \sin 2 \triangle \phi_{1}\right] \\
& =\frac{1}{2} \quad \text { and } \\
E\left[\sin ^{2} \hat{\phi}_{1}\right] & =E\left[\sin ^{2}\left(\phi_{1}+\triangle \phi_{1}\right)\right] \\
& =E\left[\frac{1}{2}\left(1-\cos \left(2 \phi_{1}+2 \triangle \phi_{1}\right)\right]\right. \\
& =\frac{1}{2}-E\left[\cos 2 \phi_{1} \cos 2 \triangle \phi_{1}-\sin 2 \phi_{1} \sin 2 \triangle \phi_{1}\right] \\
& =\frac{1}{2}, \quad \text { respectively }
\end{aligned}
$$

as $E\left[\cos 2 \phi_{1}\right]=E\left[\sin 2 \phi_{1}\right]=0$.

\section{APPENDIX C \\ SIMPLIFICATION OF RESIDUAL MAI EXPRESSION}

We need to express the residual MAI term in such a way that the average probability of bit error can be analytically determined. This then allows us to compare the analytical results of imperfect channel estimation with the simulation results. The residual MAI term $E_{s, 2}$ needs to be expressed as a function of 


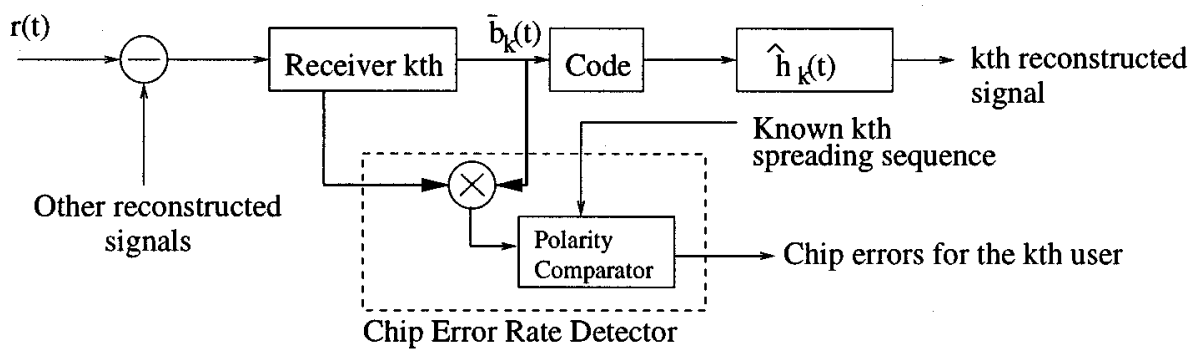

Fig. 27. Simplified block diagram of chip error rate detector.

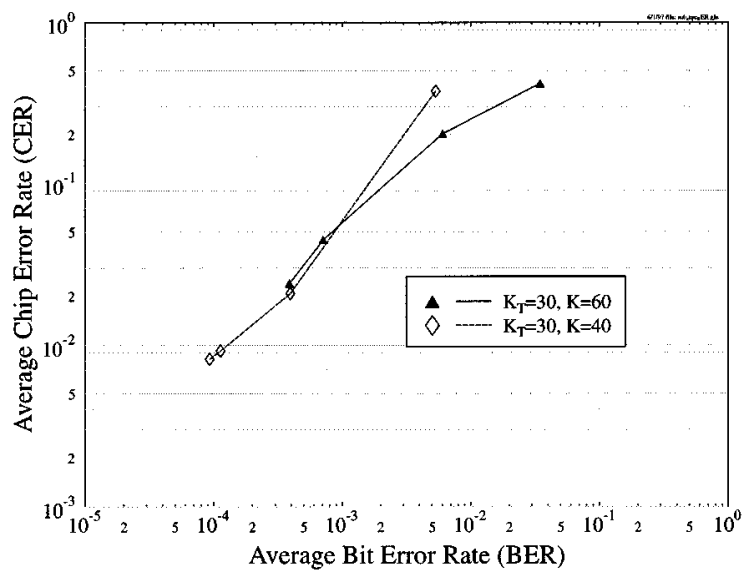

Fig. 28. Average chip error rates (CER) versus average BER for $K=40$ and 60 with perfect ranking, channel estimation, and without AWGN.

both the channel estimation and propagation error terms. Accordingly, we proceed as follows:

$$
\begin{aligned}
E_{s, 2} & =\alpha_{1} \sqrt{\frac{P}{2}} \int_{0}^{T} b_{1}\left(t-\tau_{1}\right) c_{1}\left(t-\tau_{1}\right) c_{2}(t) \cos \left(\phi_{1}-\hat{\phi}_{2}\right) d t \\
& -\left(\alpha_{1}+\triangle \alpha_{1}\right) \sqrt{\frac{P}{2}} \int_{0}^{T} \bar{b}_{1}\left(t-\tau_{1}\right) c_{1}\left(t-\tau_{1}\right) c_{2}(t) \\
& \cdot \cos \left(\hat{\phi}_{1}-\hat{\phi}_{2}\right) d t \\
= & \alpha_{1} \sqrt{\frac{P}{2}} \int_{0}^{T} b_{1}\left(t-\tau_{1}\right) c_{1}\left(t-\tau_{1}\right) c_{2}(t) \cos \left(\phi_{1}-\hat{\phi}_{2}\right) d t \\
& -\alpha_{1} \sqrt{\frac{P}{2}} \int_{0}^{T} \bar{b}_{1}\left(t-\tau_{1}\right) c_{1}\left(t-\tau_{1}\right) c_{2}(t) \cos \left(\hat{\phi}_{1}-\hat{\phi}_{2}\right) d t \\
& -\Delta \alpha_{1} \sqrt{\frac{P}{2}} \int_{0}^{T} \bar{b}_{1}\left(t-\tau_{1}\right) c_{1}\left(t-\tau_{1}\right) c_{2}(t) \cos \left(\hat{\phi}_{1}-\hat{\phi}_{2}\right) .
\end{aligned}
$$

To simply the second term in (67), we employ the power series expansion to obtain an approximate expression of $\cos \left(\hat{\phi}_{1}-\hat{\phi}_{2}\right)$. We know that the power series expansion of $\cos ((a+b)-c)$ with respect to the parameter $b$ is as follows:

$$
\begin{aligned}
= & \cos (a-c)-\sin (a-c) b-\frac{1}{2} \cos (a-c) b^{2} \\
& +\frac{1}{6} \sin (a-c) b^{3}+\frac{1}{24} \cos (a-c) b^{4}-\frac{1}{120} \sin a-c b^{5} \\
& +O\left(b^{6}\right)
\end{aligned}
$$

where $O(x)$ refers to order. Applying the series expansion as in (68) to $\cos \left(\hat{\phi}_{1}-\hat{\phi}_{2}\right)$, and ignoring the $b$ terms with power of two or more as they are negligible even for a large mean square error of 0.01 in the channel estimation errors, we have

$\cos \left(\left(\phi_{1}+\triangle \phi_{1}\right)-\hat{\phi}_{2}\right) \approx \cos \left(\phi_{1}-\hat{\phi}_{2}\right)-\sin \left(\phi_{1}-\hat{\phi}_{2}\right) \triangle \phi_{1}$.

and by substituting (69) into (67) yields

$$
\begin{aligned}
& E_{s, 2} \\
& \approx \alpha_{1} \sqrt{\frac{P}{2}} \int_{0}^{T} \breve{b}_{1}\left(t-\tau_{1}\right) c_{1}\left(t-\tau_{1}\right) c_{2}(t) \cos \left(\phi_{1}-\hat{\phi}_{2}\right) d t \\
& \quad+\triangle \phi_{1} \sqrt{\frac{P}{2}} \int_{0}^{T} \bar{b}_{1}\left(t-\tau_{1}\right) c_{1}\left(t-\tau_{1}\right) c_{2}(t) \sin \left(\phi_{1}-\hat{\phi}_{2}\right) d t \\
& \quad-\triangle \alpha_{1} \sqrt{\frac{P}{2}} \int_{0}^{T} \bar{b}_{1}\left(t-\tau_{1}\right) c_{1}\left(t-\tau_{1}\right) c_{2}(t) \cos \left(\hat{\phi}_{1}-\hat{\phi}_{2}\right) d t
\end{aligned}
$$

where $\stackrel{\vee}{b}_{1}(t)=b_{1}(t)-\bar{b}_{1}(t)$.

\section{APPENDIX D}

\section{VARIANCE OF RESIDUAL MAI}

Writing the expression for $E_{s, 2}$ as $\epsilon-\rho-\mu$, where the meaning of $\epsilon$ and $\mu$ is apparent from (70), and as $E\left[E_{s, 2}\right]=0$ because $E\left[\cos \left(\phi_{1}-\hat{\phi}_{2}\right)\right]=E\left[\triangle \alpha_{1}\right]=E\left[\triangle \phi_{1}\right]=0$, the variance of the residual MAI term, using Gaussian approximation [19], is

$$
\operatorname{Var}\left[E_{s, 2}\right]=E\left[\epsilon^{2}\right]+E\left[\rho^{2}\right]+E\left[\mu^{2}\right]
$$

where

$$
\begin{aligned}
E\left[\epsilon^{2}\right] & \\
= & E\left[\phi_{1} \alpha_{1}^{2} \frac{P}{2}\left(\int_{0}^{T} \breve{b}_{1}\left(t-\tau_{1}\right) c_{1}\left(t-\tau_{1}\right) c_{2}(t) \cos \left(\phi_{1}-\hat{\phi}_{2}\right) d t\right)^{2}\right] \\
= & E\left[\alpha_{1}^{2}\right] \frac{P}{2} E\left[\left(\breve{b}_{1}\left(t-\tau_{1}\right)\right)^{2}\right] \\
& \times E\left[\left(\int_{0}^{T} c_{1}\left(t-\tau_{1}\right) c_{2}(t) \cos \left(\phi_{1}-\hat{\phi}_{2}\right) d t\right)^{2}\right] \\
= & E\left[\alpha_{1}^{2}\right] \frac{P}{2}\left(4 P b_{s, 1}\right) T^{2} 2\left(\frac{1}{3 N}\right) E\left[\cos ^{2}\left(\phi_{1}-\hat{\phi}_{2}\right)\right] \\
= & E\left[\alpha_{1}^{2}\right] 4 P b_{s, 1} P T^{2} \frac{1}{3 N} \frac{1}{2} \\
= & \frac{T^{2}}{6 N} P E\left[\alpha_{1}^{2}\right]\left(4 P b_{s, 1}\right)
\end{aligned}
$$




$$
\begin{aligned}
E & {\left[\rho^{2}\right] } \\
= & E\left[\triangle^{2} \phi_{1} \frac{P}{2}\left(\int_{0}^{T} \bar{b}_{1}\left(t-\tau_{1}\right) c_{1}\left(t-\tau_{1}\right) c_{2}(t) \sin \left(\phi_{1}-\hat{\phi}_{2}\right) d t\right)^{2}\right] \\
= & E\left[\triangle^{2} \phi_{1}\right] \frac{P}{2} E\left[\left(\bar{b}_{1}\left(t-\tau_{1}\right)\right)^{2}\right] \\
& \times E\left[\left(\int_{0}^{T} c_{1}\left(t-\tau_{1}\right) c_{2}(t) \sin \left(\phi_{1}-\hat{\phi}_{2}\right) d t\right)^{2}\right] \\
= & \operatorname{Var}\left[\triangle \phi_{1}\right] \frac{P}{2} T^{2}(1) 2\left(\frac{1}{3 N}\right) E\left[\sin ^{2}\left(\phi_{1}-\hat{\phi}_{2}\right)\right] \\
= & \sigma_{\triangle \phi_{1}}^{2} P T^{2} \frac{1}{3 N} \frac{1}{2} \\
= & \sigma_{\triangle \phi_{1}}^{2} \frac{T^{2}}{6 N} P
\end{aligned}
$$

and

$$
\begin{aligned}
E\left[\mu^{2}\right] & E\left[\triangle^{2} \alpha_{1} \frac{P}{2}\left(\int_{0}^{T} \bar{b}_{1}\left(t-\tau_{1}\right) c_{1}\left(t-\tau_{1}\right) c_{2}(t) \cos \left(\hat{\phi}_{1}-\hat{\phi}_{2}\right) d t\right)^{2}\right] \\
= & E\left[\triangle^{2} \phi_{1}\right] \frac{P}{2} E\left[\left(\bar{b}_{1}\left(t-\tau_{1}\right)\right)^{2}\right] \\
& \times E\left[\left(\int_{0}^{T} c_{1}\left(t-\tau_{1}\right) c_{2}(t) \cos \left(\hat{\phi}_{1}-\hat{\phi}_{2}\right) d t\right)^{2}\right] \\
= & \operatorname{Var}\left[\triangle \alpha_{1}\right] \frac{P}{2} T^{2}(1) 2\left(\frac{1}{3 N}\right) E\left[\cos ^{2}\left(\hat{\phi}_{1}-\hat{\phi}_{2}\right)\right] \\
= & \sigma_{\triangle \alpha_{1}}^{2} P T^{2} \frac{1}{3 N} \frac{1}{2} \\
= & \sigma_{\triangle \alpha_{1}}^{2} \frac{T^{2}}{6 N} P
\end{aligned}
$$

where $P b_{s, 1}$ is the average probability of a bit error at the output of the first MF in the SSC stage and is defined in (19) and $E\left[\cos ^{2}\left(\phi_{1}-\hat{\phi}_{2}\right)\right]=E\left[\cos ^{2}\left(\hat{\phi}_{1}-\hat{\phi}_{2}\right)\right]=E\left[\sin ^{2}\left(\phi_{1}-\hat{\phi}_{2}\right)\right]=\frac{1}{2}$. The second moment of $b(t)$ and $\bar{b}(t)$ are obtained as follows. For the case when there is a bit error we will have $\bar{b}_{n}^{1}=-b_{n}^{1}$ so that

$$
P\left(\stackrel{\vee}{b}_{n}^{1}=2 b_{n}^{1}\right)=P b_{s, 1}
$$

where $b_{n}^{1}=b_{n}^{1}-\bar{b}_{n}^{1}$ and as $b_{1}$ is equally distributed we have

$$
P\left(\breve{b}_{n}^{1}=2\right)=P\left(\mathfrak{b}_{n}^{1}=-2\right)=\frac{P b_{s, 1}}{2} .
$$

Therefore

$$
\begin{aligned}
E\left[\left(b_{n}^{1}\right)^{2}\right] & =(2)^{2} \frac{P b_{s, 1}}{2}+(-2)^{2} \frac{P b_{s, 1}}{2} \\
& =4 P b_{s, 1} .
\end{aligned}
$$

To obtain the second moment of $\bar{b}_{1}(t)$, we know that

$$
\begin{aligned}
E\left[\left(\bar{b}_{n}^{1}\right)^{2}\right] & =(1)^{2} P b_{s, 1}+(-1)^{2}\left(1-P b_{s, 1}\right) \\
& =(1)^{2}\left(1-P b_{s, 1}\right)+(-1)^{2} P b_{s, 1} \\
& =1
\end{aligned}
$$

Similarly, we need to find the expectation of $\sin ^{2}\left(\phi_{1}-\hat{\phi}_{2}\right)$ and $\cos ^{2}\left(\hat{\phi}_{1}-\hat{\phi}_{2}\right)$ in order to evaluate the variance of the residual MAI found in Appendix D. They are as follows:

$$
\begin{aligned}
E\left[\sin ^{2}\left(\phi_{1}-\hat{\phi}_{2}\right)\right] & =E\left[\left(\sin \phi_{1} \cos \hat{\phi}_{2}-\cos \phi_{1} \sin \hat{\phi}_{2}\right)^{2}\right] \\
& =E\left[\sin ^{2} \phi_{1} \cos ^{2} \hat{\phi}_{2}\right]+E\left[\cos ^{2} \phi_{1} \sin ^{2} \hat{\phi}_{2}\right] \\
& =\frac{1}{2}
\end{aligned}
$$

as $E\left[\sin \phi_{1}\right]=0, E\left[\sin ^{2} \phi_{1}\right]=E\left[\cos ^{2} \hat{\phi}_{2}\right]=\frac{1}{2}$ and $E\left[\cos ^{2} \phi_{1}\right]=E\left[\sin ^{2} \hat{\phi}_{2}\right]=\frac{1}{2}$ and

$$
\begin{aligned}
E\left[\cos ^{2}\left(\hat{\phi}_{1}-\hat{\phi}_{2}\right)\right] & =E\left[\cos ^{2} \hat{\phi}_{1} \cos ^{2} \hat{\phi}_{2}+\sin ^{2} \hat{\phi}_{1} \sin ^{2} \hat{\phi}_{2}\right] \\
& =E\left[\cos ^{2} \hat{\phi}_{1} \cos ^{2} \hat{\phi}_{2}\right]+E\left[\sin ^{2} \hat{\phi}_{1} \sin ^{2} \hat{\phi}_{2}\right] \\
& =\frac{1}{2}
\end{aligned}
$$

as $E\left[\cos ^{2} \hat{\phi}_{1}\right]=E\left[\cos ^{2} \hat{\phi}_{2}\right]=\frac{1}{2}$ and $E\left[\sin ^{2} \hat{\phi}_{1}\right]=$ $E\left[\sin ^{2} \hat{\phi}_{2}\right]=\frac{1}{2}$.

\section{ACKNOWLEDGMENT}

The authors would like to thank Dr. G. Povey of the Department of Electrical Engineering, University of Edinburgh, Edinburgh, U.K., for the use of the Rayleigh fading simulator.

\section{REFERENCES}

[1] J. Laster and J. Reed, "A survey of adaptive single channel inteference rejection techniques for wireless communications," in Proc. Virginia Tech. 4th Symp. Wireless Personal Communications, June 1994, pp. 571-575.

[2] R. Kohno, P. Rapajic, and B. Vucetic, "An overview of adaptive techniques for interference minimization in CDMA systems," Wireless Personal Commun., vol. 1, no. 1, pp. 3-21, 1994.

[3] S. Verdu, "Minimum probability of error for asynchronous Gaussian multiple access channels," IEEE Trans. Inform. Theory, vol. 32, no. 1, pp. 85-96, 1986.

[4] P. R. Patel and J. Holtzman, "Analysis of a DS/CDMA successive interference cancellation scheme using correlations," in Proc. IEEE Globecom'93, pp. 76-80.

[5] J. Holtzman, "DS/CDMA successive interference cancelation," in Proc. ISSSTA'94, vol. 7, 1994, pp. 69-78.

[6] A. Viterbi, "Very low rate convolutional codes for maximum theoretical performance of spread-spectrum multiple access channels," IEEE J. Select Areas Commun., vol. 8, no. 4, pp. 641-649, 1990.

[7] S. Striglis, N. Y. A. Kaul, and B. Woerner, "A multistage rake receiver for improved capacity in CDMA systems," in Proc. VTC'94, Stockholm, Sweden, 1994, pp. 789-393.

[8] A. Kaul and B. D. Woerner, "An analysis of adaptive multistage interference cancellation for CDMA," in Proc. VTC'95, Chicago, IL, May 1995, pp. 82-86.

[9] Y. Li and R. Steele, "Serial interference cancellation method for CDMA," Electron. Lett., vol. 30, no. 19, pp. 1581-1583, 1994.

[10] P. Patel and J. Holtzman, "Performance comparison of a DS/CDMA system using a succesive interference cancellation (IC) scheme and a parallel IC scheme under fading," in Proc. ICC'94, New Orleans, LA, May 1994, pp. 510-514.

[11] T.-B. Oon, R. Steele, and Y. Li, "Cancellation frame size for a quasi single bit detector in asynchronous CDMA channel," IEE Electron. Lett., vol. 33, no. 4, pp. 258-259, 1997.

[12] J. G. Proakis, Digital Communications. New York: McGraw-Hill, 1989. 
[13] Y. Yoon, R. Kohno, and H. Imai, "A spread-spectrum multiaccess system with cochannel inteference cancelllation for multipath fading channels," IEEE J. Select Areas Commun., vol. 11, no. 7, pp. 1067-1075, 1993.

[14] M. Pursley, "Performance evaluation for phase-coded spread-spectrum multiple acccess communication-Part I: System analysis," IEEE Trans. Commun., vol. 25, no. 8, pp. 800-803, 1977.

[15] G. Korn and T. Korn, Mathematical Handbook For Scientists and Engineers. New York: McGraw-Hill, 1977.

[16] H. A. David, Order Statistics. New York: Wiley, 1981, pp. 8-13.

[17] S. Tachikawa, "Performance of $M$-ary/spread spectrum multiple access communication systems using co-channel interference cancellation techniques," in Proc. IEEE ISSSTA'92, vol. 11, Yokohama, Japan, pp. 4-5.

[18] R. Prasad, CDMA for Wirless Personal Communications. Norwood, MA: Artech House, 1996, pp. 316-326.

[19] A. Lam and S. Tantaratana, Theory and Applications of Spread Spectrum Systems. New York: IEEE, 1994.

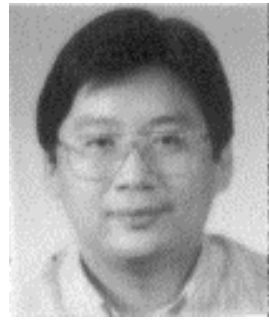

Tik-Bin Oon (M'92) received the B.E. degree (Hon. class 1) in electrical engineering from the University of New South Wales, Sydney, Australia, in 1991 and the Ph.D. degree from the University of Southampton, U.K., in 1998.

Prior to attaining the B.E. degree, he was an Associate Engineer at CET Technologies, Singapore, in the area of reliability engineering. After completing his studies in 1991, he returned to work as a Development Engineer. From 1992 to 1994, he was in charge of the development of tactical data terminal and GPSrelated systems. His research interests are in CDMA multiuser detection and cancellation schemes, adaptive antenna techniques, channel estimations, and mobile communications in general.

Dr. Oon received the Singapore Technologies Group scholarship in 1989 to pursue a B.E. degree. In 1994, he received the ORS scholarship from CCVP, U.K., and a Singapore Technologies Group scholarship to pursue the Ph.D. degree at the Communication Research Group of the Department of Electronics and Computer Science, University of Southampton. He is a member of the Royal Institute of Navigation and an Associate Member of the IEE.

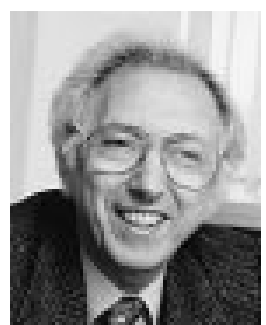

Raymond Steele (F'96) received the B.Sc. degree in electrical engineering from Durham University, U.K., in 1959 and the Ph.D. and D.Sc. degrees from Loughborough University of Technology, U.K., in 1975 and 1983 , respectively.

He is the Chairman of Multiple Access Communications Ltd., a company concerned with digital mobile radio systems, and Head of the Communications Research Group in the Department of Electronics and Computer Science at Southampton University, Southampton, U.K. Before receiving the B.Sc. degree, he was an indentured Apprentice Radio Engineer. After research and development posts with E. K. Cole, Cossor Radar and Electronics, and Marconi, he joined the lecturing staff at the Royal Naval College, London, U.K. He moved to Loughborough University in 1968 where he lectured and directed a research group in digital encoding of speech and picture signals. During the summers of 1975, 1977, and 1978, he was a Consultant to the Acoustics Research Department at Bell Laboratories in the United States, and in 1979 he joined the company's Communications Methods Research Department, Crawford Hill Laboratory, NJ. He returned to England in 1983 to become Professor of Communications in the Department of Electronics and Computer Science, University of Southampton. From 1983 to 1986, he was a nonexecutive Director of Plessey Research and Technology and from 1983 to 1989 a Consultant to British Telecom Research Laboratories. In 1986, he formed Multiple Access Communications Ltd. He is the author of Delta Modulation Systems (New York: Halsted, 1975), Editor of Mobile Radio Communications (New York: IEEE Press, 1992), coauthor of Source-Matched Mobile Communications (New York: IEEE Press, 1995), and author of over 200 technical publications. He is a Member of the Advisory Board of IEEE Personal Communications (the magazine of nomadic communications and computing).

Dr. Steele and his coauthors were awarded the Marconi Premium in 1979 and 1989 as well as the Bell System Technical Journal's Best Mathematics, Communications, Techniques, Computing and Software, and Social Sciences Paper in 1981. He has been a conference and session organizer of numerous international conferences and a keynote speaker at many international meetings. He is a Member of the Advisory Board of the International Journal of Wireless Information Networks. He is a Fellow of the Royal Academy of Engineering and the IEE and a member of the IEEE Avant Garde.

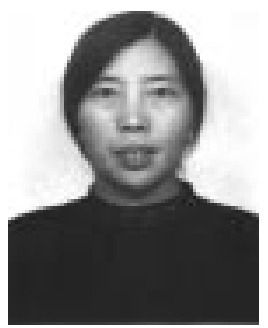

Ying $\mathbf{L i}$ received the B.Sc. degree from the University of Taiyuan Industry, China, in 1982 and the M.Sc and $\mathrm{Ph} . \mathrm{D}$ degrees from Xidian University, China, in 1986 and 1989, respectively, all in information engineering.

From 1989 to 1994, she was a Lecturer and then an Associate Professor of Information Engineering at Xidian University, where she was engaged in research, design, and development of mobile radio communications systems. From 1995 to 1997 , she was a Research Fellow in the Communication Research Group of the Department of Electronics and Computer Science, University of Southampton, U.K., working on a campus microcellular network testbed. Since October 1997, she has been with Multiple Access Communication Limited, Southampton, U.K., where she is involved in the software design and implementation of the layer-two and layer-three protocols in the GSM mobile phone. She has also performed some consultancy work related to mobile radio networks. Her current professional interests are mobile communication network structures, network protocols, and radio resource control algorithms. 